\title{
Sequence-Subset Distance and Coding for Error Control in DNA-based Data Storage
}

\author{
Wentu Song, Kui Cai, senior member, IEEE and Kees A. Schouhamer Immink, Fellow, IEEE
}

\begin{abstract}
The process of DNA-based data storage (DNA storage for short) can be mathematically modelled as a communication channel, termed DNA storage channel, whose inputs and outputs are sets of unordered sequences. To design error correcting codes for DNA storage channel, a new metric, termed the sequence-subset distance, is introduced, which generalizes the Hamming distance to a distance function defined between any two sets of unordered vectors and helps to establish a uniform framework to design error correcting codes for DNA storage channel. We further introduce a family of error correcting codes, referred to as sequence-subset codes, for DNA storage and show that the error-correcting ability of such codes is completely determined by their minimum distance. We derive some upper bounds on the size of the sequence-subset codes including a tight bound for a special case, a Singleton-like bound and a Plotkinlike bound. We also propose some constructions, including an optimal construction for that special case, which imply lower bounds on the size of such codes.
\end{abstract}

Index Terms-DNA data storage, error-correcting codes, Singleton bound, Plotkin bound.

\section{INTRODUCTION}

The idea of storing data in synthetic DNA strands (sequences) has been around since 1988 [1] and DNA-based data storage has been progressing rapidly in recent years with the development of DNA synthesis and sequencing technology. Compared to traditional magnetic and optical media, DNA storage has some competing advantages such as extreme high density, long durability [10], and low energy consumption [2].

A DNA strand is mathematically represented by a quaternary sequence, each symbol represents one of the four types of base nucleotides: adenine (A), cytosine (C), guanine (G) and thymine $(\mathrm{T})$. Basically, in a DNA-based storage system, the original binary data is first encoded to a set of quaternary sequences. Then the corresponding DNA strands are synthesized and stored in DNA pools. To retrieve (read) the original data, the stored DNA strands are sequenced to generate a set of quaternary sequences, which are then decoded to the original binary data. The process of DNA synthesizing, storing and sequencing can be mathematically modelled as a communication channel, called the DNA storage channel, which can be depicted by Fig. 1.

The sequencing process can be modelled as a randomly sampling and reading of molecules with replacement from the DNA pool [16]. Some DNA strands may have many

Wentu Song and Kui Cai are with Singapore University of Technology and Design, Singapore, e-mail: \{wentu_song, cai_kui\}@sutd.edu.sg.

Kees A. Schouhamer Immink is with Turing Machines Inc, Willemskade 15d, 3016 DK Rotterdam, The Netherlands, e-mails: immink@turingmachines.com. copies that are sequenced while some strands may never be sequenced. Moreover, since the synthesis/sequencing process is prone to errors, a specific DNA strand in the pool may have many noisy copies that are contained in the sequencing output. These sequenced strands are clustered according to their Levenshtein distance or by some other methods (e.g., see [3], [4]), and then the clustered sequences are reconstructed by performing an estimate for each cluster [5]. All different estimated sequences form the output of the DNA storage channel. Another characteristic of DNA storage channel is that unlike the conventional magnetic or optical recording systems, the DNA sequences are stored in "pools", where structured addressing is not allowed. Therefore, the inputs and outputs of the DNA storage channel can be viewed as sets of unordered DNA sequences.

The output of the DNA storage channel may be distorted by the following five types of errors:

- Sequence deletion: One or more of the input sequences are lost. A DNA strand is lost if it is never sequenced. Another case of sequence deletion is when there are $t(>1)$ strands that are changed to the same strand by substitution errors, then any $t-1$ of them are viewed as lost sequences. As a result, the number of output sequences is smaller than the number of input sequences.

- Sequence insertion: One or more sequences that do not belong to the set of input sequences are added into the output sequences. If the output of the channel contains $t(>1)$ different noisy copies of an input sequence, then any $t-1$ of them can be viewed as inserted sequences. As a result, the number of output sequences is larger than the number of input sequences.

- Symbol deletion: One or more symbols in a sequence are removed. As a result, the length of the erroneous sequence is decreased.

- Symbol insertion: One or more symbols are added into a sequence. As a result, the length of the erroneous sequence is increased.

- Symbol substitution: One or more symbols in a sequence are replaced by other symbols. In this case, the length of the erroneous sequence remains unchanged.

Note that sequence deletion and sequence insertion can take place simultaneously. If the number of sequence deletions equals the number of sequence insertions, then the total number of input sequences remain unchanged.

To combat different types of errors in DNA synthesizing and sequencing, various coding techniques are used by DNA storage. Most demonstration research works employ constrained 


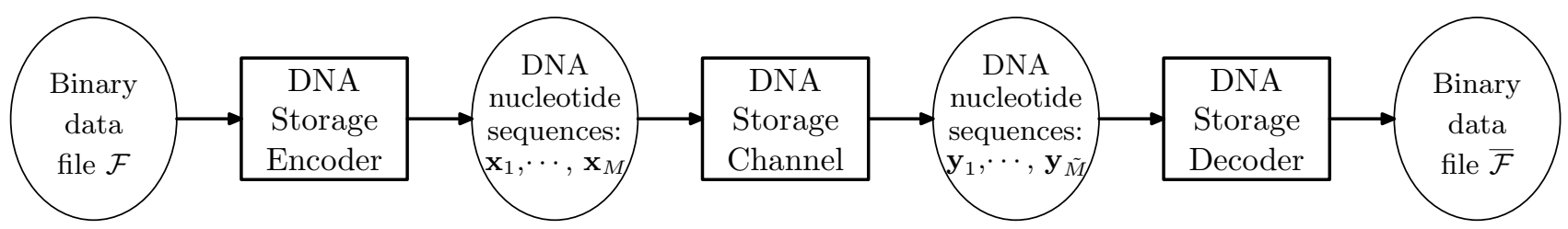

Fig 1. System model of the DNA storage: The DNA storage channel is the mathematical model of the process of DNA synthesizing, storing and sequencing. A reliable system should guarantee that with sufficiently high probability the decoded file $\overline{\mathcal{F}}$ equals to the original file $\mathcal{F}$.

coding combined with classical error correcting codes (e.g. Reed-Solomon codes) [2]-[13]. In addition, to combat the lack of ordering of the transmitted sequences, a unique address (index) is added to each sequence.

Codes that can correct $s$ (or fewer) losses of sequences and $e$ (or fewer) substitutions in each of $t$ (or fewer) sequences were studied in [14] by considering the so-called error ball. Codes dealing with insertion/deletion errors were also studied in [14]. Codes that can correct a total of $K$ substitution errors were studied in [15].

\section{A. Our Contribution}

In this paper, we consider error control for DNA storage channel by introducing a new metric, termed the sequencesubset distance, over the power set of the set of all vectors of fixed length over a finite alphabet, which is the space of the inputs/outputs of the DNA storage channel. This metric is a generalization of the classical Hamming distance and can help to establish a uniform framework to design codes for DNA storage channel that can correct errors of sequence deletion, sequence insertion and symbol substitution.

We study error correcting codes with respect to the sequence-subset distance, which we refer to as sequencesubset codes, for DNA-based data storage. We show that similar to codes with respect to the classical Hamming distance, a sequence-subset code $\mathcal{C}$ can correct any number of $n_{\mathrm{D}}$ sequence deletions, $n_{\mathrm{I}}$ sequence insertions, and totally $n_{\mathrm{S}}$ symbol substitutions, provided that $n_{\mathrm{S}}+L \cdot \max \left\{n_{\mathrm{I}}, n_{\mathrm{D}}\right\} \leq$ $\frac{d_{\mathrm{S}}(\mathcal{C})-1}{2}$, where $L$ is the length of the sequences and $d_{\mathrm{S}}(\mathcal{C})$ is the minimum distance of $\mathcal{C}$.

We derive some upper bounds on the size of the sequencesubset codes including a tight bound for the special case that $d=L M$, a Singleton-like bound and a Plotkin-like bound, where $M$ is the codeword size (i.e., the number of sequences in each codeword of the sequence-subset codes).

We give a construction of optimal codes (with respect to size) for the special case that $d=L M$ and $M^{\frac{1}{L}}$ is an integer, where $d$ is the minimum distance of the code. We also give some general constructions of sequence-subset codes, which imply lower bounds of the size of such codes.

\section{B. Related Work}

The similar channel model for DNA storage was also studied in [14], [15] and [16].

In [14] and [15], data is stored in an unordered set of $M$ strings of length $L$ (the input of the DNA storage channel), where $M$ and $L$ are some fixed positive integers. The work of [14] considered the error-correcting problem by restricting that $s$ sequences are lost during the synthesizing/sequencing process and the output of the channel is a subset of $M-s$ input sequences, among which $M-s-t$ sequences are correctly reconstructed and $t$ sequences are reconstructed with errors such that each sequence has at most $\epsilon$ errors, where possible errors are symbol insertion/deletion and/or substitution. In [15], the channel was studied under the assumption that the values of a total of $K$ different positions in the $M$ input sequences are changed (i.e., there are totally $K$ symbol substitutions). Since the erroneous sequence may be equal to another existing sequence, which in fact induces sequence deletion, the output of the channel is a set of $T$ strings of length $L$ for some $T$ such that $M-K \leq T \leq M$.

In [16], DNA storage is modelled as a channel whose inputs are multisets of $M$ sequences of length $L$ while the output of the channel is a multiset of $N$ sequences of length $L$, which is obtained by drawing $N$ samples independently and uniformly at random, with replacement, from the $M$ input sequences, where $M, L, N$ are the fixed parameters of the channel. It also assumes that each sampled molecule is read error-free.

Comparison of our model with the models of [14], [15] and [16] is given in Table 1.

Another model for DNA storage channel, which focuses on modelling the process of synthesis and sequencing of single DNA strand, was consider in [17]. Different from our model, the input of this channel is a single DNA sequence (rather than a set of sequences), and through the process of synthesis and sequencing, a set of DNA fragments along with their frequency count is obtained, which can be represented by a profile vector. Three types of errors, namely, substitution errors due to synthesis, coverage errors, and $\ell$-gram substitution errors due to sequencing, are considered in [17].

There are still some other communication channels similar to DNA storage channel. The permutation channel considered in [18] has input and output as vectors over a finite alphabet and the transmitted vector is corrupted by a permutation on its coordination. The permutation channel with impairments was considered in [19], where the input and output are multisets, rather than vectors, of symbols from a finite alphabet. Unlike the DNA storage channel, the structure information of the sequences (i.e., the Hamming distance between sequences when the sequence length $L>1$ ) is not considered in such models.

\section{Organization}

The rest of the paper is organized as follows. In Section II, we introduce the sequence-subset distance and provide the 


\begin{tabular}{|l|l|l|l|}
\hline \multicolumn{1}{|c|}{ Model } & \multicolumn{1}{|c|}{ Inputs } & \multicolumn{1}{c|}{ Outputs } & \multicolumn{1}{c|}{ Error types } \\
\hline Model of [14] & $\begin{array}{l}\text { A set of } M \text { DNA sequences of length } \\
L, \text { for some fixed } M \text { and } L\end{array}$ & $\begin{array}{l}\text { A set of } M-s \text { DNA sequences with } \\
\text { at most } t \text { erroneous sequences }\end{array}$ & $\begin{array}{l}\text { Sequence deletion, symbol insertion, } \\
\text { deletion and substitution }\end{array}$ \\
\hline Model of [15] & $\begin{array}{l}\text { A set of } M \text { DNA sequences of length } \\
L, \text { for some fixed } M \text { and } L\end{array}$ & $\begin{array}{l}\text { A set of } T \text { DNA sequences with } \\
\text { totally } K \text { substitutions, where } M- \\
K \leq T \leq M\end{array}$ & $\begin{array}{l}\text { Symbol substitution, sequence dele- } \\
\text { tion induced by symbol substitution }\end{array}$ \\
\hline Model of [16] & $\begin{array}{l}\text { A multi-set of } M \text { DNA sequences of } \\
\text { length } L \text { for some fixed } M \text { and } L\end{array}$ & $\begin{array}{l}\text { A multi-set of } N \text { DNA sequences } \\
\text { drawn randomly with replacement } \\
\text { from the } M \text { input sequences }\end{array}$ & Sequence deletion \\
\hline Our Model & $\begin{array}{l}\text { A set of } M \text { DNA sequences of length } \\
L, \text { where } L \text { is fixed but } M \text { is not } \\
\text { necessarily fixed }\end{array}$ & $\begin{array}{l}\text { A set of } \tilde{M} \text { DNA sequences, which } \\
\text { mayclude erroneous sequences and } \\
\text { additional inserted sequences }\end{array}$ & $\begin{array}{l}\text { Sequence deletion, sequence inser- } \\
\text { tion and symbol substitution }\end{array}$ \\
\hline
\end{tabular}

Table 1. Comparison of different models for DNA storage channel.

basic properties of codes with sequence-subset distance. We analyze the upper bound on the size of sequence-subset codes in Section III and give some constructions of such codes in Section IV. The paper is concluded in Section V.

\section{Notations}

The following notations will be used in this paper:

1) For any positive integer $n,[n]:=\{1,2, \cdots, n\}$.

2) For any set $\mathbb{A},|\mathbb{A}|$ denotes the size (i.e., cardinality) of $\mathbb{A}$ and $\mathcal{P}(\mathbb{A})$ denotes the power set of $\mathbb{A}$ (i.e., the collection of all subsets of $\mathbb{A})$.

3) For any two sets $\mathbf{X}$ and $\mathbf{Y}, \mathbf{X} \backslash \mathbf{Y}$ is the set of all elements of $\mathbf{X}$ that do not belong to $\mathbf{Y}$.

4) For any $n$-tuple $\mathbf{x} \in \mathbb{A}^{n}$ and any $i \in[n], \mathbf{x}(i)$ denotes the $i$ th coordinate of $\mathbf{x}$, and hence $\mathbf{x}$ is denoted as $\mathbf{x}=$ $(\mathbf{x}(1), \mathbf{x}(2), \cdots, \mathbf{x}(n))$.

\section{PRELIMINARY}

We first introduce the concept of sequence-subset distance. Then we discuss the error pattern and error-correcting in DNA storage channel using codes with sequence-subset distance.

\section{A. Sequence-Subset Distance}

Let $\mathbb{A}$ be a fixed finite alphabet. For DNA data storage, typically $\mathbb{A}=\{A, T, C, G\}$, representing the four types of base nucleotides. In this work, for generality, we assume that $\mathbb{A}$ is any fixed finite alphabet of size $q \geq 2$.

Let $L$ be a positive integer. For any $\mathbf{x}_{1}, \mathbf{x}_{2} \in \mathbb{A}^{L}$, the Hamming distance between $\mathbf{x}_{1}$ and $\mathbf{x}_{2}$, denoted by $d_{\mathrm{H}}\left(\mathbf{x}_{1}, \mathbf{x}_{2}\right)$, is defined as the number of coordinates where $\mathbf{x}_{1}$ and $\mathbf{x}_{2}$ differ, that is,

$$
d_{\mathrm{H}}\left(\mathbf{x}_{1}, \mathbf{x}_{2}\right):=\left|\left\{i \in[L] ; \mathbf{x}_{1}(i) \neq \mathbf{x}_{2}(i)\right\}\right| .
$$

For any two subsets $\mathbf{X}_{1}$ and $\mathbf{X}_{2}$ of $\mathbb{A}^{L}$ such that $\left|\mathbf{X}_{1}\right| \leq\left|\mathbf{X}_{2}\right|$ and any injection $\chi: \mathbf{X}_{1} \rightarrow \mathbf{X}_{2}$, denote

$$
d_{\chi}\left(\mathbf{X}_{1}, \mathbf{X}_{2}\right):=\sum_{\mathbf{x} \in \mathbf{X}_{1}} d_{\mathbf{H}}(\mathbf{x}, \chi(\mathbf{x}))+L\left(\left|\mathbf{X}_{2}\right|-\left|\mathbf{X}_{1}\right|\right) .
$$

Then a natural way to generalize the Hamming distance to the space of all subsets of $\mathbb{A}^{L}$ is as follows.
Definition 1: For any $\mathbf{X}_{1}, \mathbf{X}_{2} \subseteq \mathbb{A}^{L}$, without loss of generality, assuming $\left|\mathbf{X}_{1}\right| \leq\left|\mathbf{X}_{2}\right|$, the sequence-subset distance between $\mathbf{X}_{1}$ and $\mathbf{X}_{2}$ is defined as

$$
d_{\mathrm{S}}\left(\mathbf{X}_{1}, \mathbf{X}_{2}\right)=d_{\mathrm{S}}\left(\mathbf{X}_{2}, \mathbf{X}_{1}\right):=\min _{\chi \in \mathscr{X}} d_{\chi}\left(\mathbf{X}_{1}, \mathbf{X}_{2}\right),
$$

where $\mathscr{X}$ is the set of all injections $\chi: \mathbf{X}_{1} \rightarrow \mathbf{X}_{2}$ 1]

Example 1: Suppose $\mathbb{A}=\{0,1\}$ and $L=4$. Consider $\mathbf{X}_{1}=\left\{\mathbf{x}_{1}, \mathbf{x}_{2}, \mathbf{x}_{3}\right\}$ and $\mathbf{X}_{2}=\left\{\mathbf{y}_{1}, \mathbf{y}_{2}, \mathbf{y}_{3}, \mathbf{y}_{4}\right\}$, where $\mathbf{x}_{1}=$ $1010, \mathbf{x}_{2}=0010, \mathbf{x}_{3}=1101, \mathbf{y}_{1}=1101, \mathbf{y}_{2}=0011$, $\mathbf{y}_{3}=1011$ and $\mathbf{y}_{4}=1100$. Let $\chi_{0}: \mathbf{X}_{1} \rightarrow \mathbf{X}_{2}$ be such that $\chi_{0}\left(\mathbf{x}_{1}\right)=\mathbf{y}_{3}, \chi_{0}\left(\mathbf{x}_{2}\right)=\mathbf{y}_{2}$ and $\chi_{0}\left(\mathbf{x}_{3}\right)=\mathbf{y}_{1}$. Then by (1), we can obtain $d_{\chi_{0}}\left(\mathbf{X}_{1}, \mathbf{X}_{2}\right)=6$. We can further verify that $d_{\chi}\left(\mathbf{X}_{1}, \mathbf{X}_{2}\right) \geq 6$ for all injections $\chi: \mathbf{X}_{1} \rightarrow \mathbf{X}_{2}$. Hence by (2), we have $d_{\mathrm{S}}\left(\mathbf{X}_{1}, \mathbf{X}_{2}\right)=d_{\chi_{0}}\left(\mathbf{X}_{1}, \mathbf{X}_{2}\right)=6$.

Remark 1: Given any subsets $\mathbf{X}_{1}, \mathbf{X}_{2}$ of $\mathbb{A}^{L}$ such that $\left|\mathbf{X}_{1}\right| \leq\left|\mathbf{X}_{2}\right|$, let $V_{1}=\mathbf{X}_{1} \cup V_{0}$ and $V_{2}=\mathbf{X}_{2}$, where $V_{0}$ is a set disjoint with $\mathbf{X}_{1}$. We can construct a complete bipartite weighted graph $G$ with bipartition $\left(V_{1}, V_{2}\right)$ such that for each $\mathbf{x}_{1} \in V_{1}$ and $\mathbf{x}_{2} \in V_{2}$, the weight of the edge $\left(\mathbf{x}_{1}, \mathbf{x}_{2}\right)$ is $d_{\mathrm{H}}\left(\mathbf{x}_{1}, \mathbf{x}_{2}\right)$, where we define $d_{\mathrm{H}}\left(\mathbf{x}_{1}, \mathbf{x}_{2}\right)=L$ for any $\mathbf{x}_{1} \in V_{0}$ and $\mathbf{x}_{2} \in V_{2}$. Then by Definition 1 , the sequencesubset distance between $\mathbf{X}_{1}$ and $\mathbf{X}_{2}$ can be computed from a minimum weight perfect matching of $G$, which can be done in time $O\left(\left|\mathbf{X}_{2}\right|^{3}\right)$ using the Kuhn-Munkres algorithm [22].

We now prove some important properties of the function $d_{\mathrm{S}}(\cdot, \cdot)$ and then prove that it is really a distance function.

First, intuitively, the elements in $\mathbf{X}_{1} \cap \mathbf{X}_{2}$ should have no effect on the sequence-subset distance between $\mathbf{X}_{1}$ and $\mathbf{X}_{2}$. This is shown to be true by the following lemma and corollary.

Lemma 1: For any $\mathbf{X}_{1}, \mathbf{X}_{2} \subseteq \mathbb{A}^{L}$ such that $\left|\mathbf{X}_{1}\right| \leq\left|\mathbf{X}_{2}\right|$, there exists an injection $\chi_{0} \in \mathscr{X}$ such that $d_{\mathbf{S}}\left(\mathbf{X}_{1}, \mathbf{X}_{2}\right)=$ $d_{\chi_{0}}\left(\mathbf{X}_{1}, \mathbf{X}_{2}\right)$ and $\chi_{0}(\mathbf{x})=\mathbf{x}$ for all $\mathbf{x} \in \mathbf{X}_{1} \cap \mathbf{X}_{2}$.

Proof: The proof is given in Appendix A.

Corollary 1: For any two subsets $\mathbf{X}_{1}$ and $\mathbf{X}_{2}$ of $\mathbb{A}^{L}$,

$$
d_{\mathrm{S}}\left(\mathbf{X}_{1}, \mathbf{X}_{2}\right)=d_{\mathrm{S}}\left(\mathbf{X}_{1} \backslash \mathbf{X}_{2}, \mathbf{X}_{2} \backslash \mathbf{X}_{1}\right) .
$$

Proof: This corollary is just a direct consequence of Definition 1 and Lemma 1.

Lemma 2: Suppose $\mathbf{X}_{1}, \mathbf{X}_{2} \subseteq \mathbb{A}^{L}$ such that $\left|\mathbf{X}_{1}\right| \leq\left|\mathbf{X}_{2}\right|$. Suppose $\mathbf{X}_{2}^{\prime} \subseteq \mathbf{X}_{2}$ such that $\left|\mathbf{X}_{1}\right| \leq\left|\mathbf{X}_{2}^{\prime}\right|$. Then

$$
d_{\mathrm{S}}\left(\mathbf{X}_{1}, \mathbf{X}_{2}^{\prime}\right) \leq d_{\mathrm{S}}\left(\mathbf{X}_{1}, \mathbf{X}_{2}\right) \text {. }
$$

${ }^{1} \mathrm{~A}$ more accurate notation for the set $\mathscr{X}$ is $\mathscr{X}_{\mathbf{X}_{1}, \mathbf{x}_{2}}$ because it is related to the subsets $\mathbf{X}_{1}$ and $\mathbf{X}_{2}$. However, we can omit the subscripts safely because they can be easily specified by the context. 
Proof: The proof is given in Appendix B.

Now we prove that $d_{\mathrm{S}}(\cdot, \cdot)$ is really a distance function (metric) over $\mathcal{P}\left(\mathbb{A}^{L}\right)$.

Theorem 1: The function $d_{\mathrm{S}}(\cdot, \cdot)$ is a distance function over the power set $\mathcal{P}\left(\mathbb{A}^{L}\right)$.

Proof: The proof is given in Appendix C.

\section{B. Error Pattern of DNA Storage Channel}

In this paper we consider DNA storage channel with sequence deletion/insertion and symbol substitution. The input of the channel is a set of unordered sequences

$$
\mathbf{X}=\left\{\mathbf{x}_{1}, \mathbf{x}_{2}, \cdots, \mathbf{x}_{M}\right\} \subseteq \mathbb{A}^{L}
$$

and the output is another set of unordered sequences

$$
\mathbf{Y}=\left\{\mathbf{y}_{1}, \mathbf{y}_{2}, \cdots, \mathbf{y}_{\tilde{M}}\right\} \subseteq \mathbb{A}^{L},
$$

where $L$ is the length of the sequences. Usually, $\mathbf{Y} \neq \mathbf{X}$ because of the channel noise. Sequences in the subset $\mathbf{X} \cap \mathbf{Y}$ are correctly transmitted; Sequences in $\mathbf{X} \backslash \mathbf{Y}$ are either lost (sequence deletion) or changed to sequences in $\mathbf{Y} \backslash \mathbf{X}$ (symbol substitution); Sequences in $\mathbf{Y} \backslash \mathbf{X}$ are either excessive (sequence insertion) or obtained from some sequences in $\mathbf{X} \backslash \mathbf{Y}$ (symbol substitution). Let $n_{\mathrm{I}}, n_{\mathrm{D}}$ and $n_{\mathrm{S}}$ denote the total number of sequence insertions, sequence deletions and symbol substitutions, respectively, in $\mathbf{Y}$. Then we call the 3 -tuple $\left(n_{\mathrm{I}}, n_{\mathrm{D}}, n_{\mathrm{S}}\right)$ the error pattern of Y. Furthermore, we have the following lemma.

Lemma 3: Suppose the channel input is $\mathbf{X}$ and output is $\mathbf{Y}$. If the error pattern of $\mathbf{Y}$ is $\left(n_{\mathrm{I}}, n_{\mathrm{D}}, n_{\mathrm{S}}\right)$, then

$$
d_{\mathrm{S}}(\mathbf{X}, \mathbf{Y}) \leq n_{\mathrm{S}}+L \cdot \max \left\{n_{\mathrm{I}}, n_{\mathrm{D}}\right\}
$$

Proof: Note that we can always partition the two subsets $\mathbf{X} \backslash \mathbf{Y}$ and $\mathbf{Y} \backslash \mathbf{X}$ as

$$
\mathbf{X} \backslash \mathbf{Y}=\mathbf{X}_{\mathrm{D}} \cup \mathbf{X}_{\mathrm{S}} \quad \text { and } \quad \mathbf{Y} \backslash \mathbf{X}=\mathbf{Y}_{\mathrm{I}} \cup \mathbf{Y}_{\mathrm{S}},
$$

where $\mathbf{X}_{\mathrm{D}}$ is the set of lost input sequences, $\mathbf{X}_{\mathrm{S}}$ is the set of input sequences that are changed to $\mathbf{Y}_{\mathrm{S}}$ by symbol substitution, and $\mathbf{Y}_{\mathrm{I}}$ is the set of sequences that are inserted to $\mathbf{Y}$. Clearly, we have

$$
n_{\mathrm{I}}=\left|\mathbf{Y}_{\mathrm{I}}\right| \quad \text { and } \quad n_{\mathrm{D}}=\left|\mathbf{X}_{\mathrm{D}}\right| .
$$

Moreover, $\left|\mathbf{X}_{\mathrm{S}}\right|=\left|\mathbf{Y}_{\mathrm{S}}\right|$ and there exists a bijection $\chi: \mathbf{X}_{\mathrm{S}} \rightarrow$ $\mathbf{Y}_{\mathrm{S}}$ such that for each $\mathbf{x} \in \mathbf{X}_{\mathrm{S}}, \chi(\mathbf{x})$ is the erroneous sequence of $\mathbf{x}$ by symbol substitution. Hence, we have

$$
n_{\mathrm{S}}=\sum_{\mathbf{x} \in \mathbf{X}_{\mathrm{S}}} d_{\mathrm{H}}(\mathbf{x}, \chi(\mathbf{x})) .
$$

For further discussion, we need to consider the following two cases.

Case 1: $n_{\mathrm{I}} \leq n_{\mathrm{D}}$. In this case, we have $\left|\mathbf{Y}_{\mathrm{I}}\right|=n_{\mathrm{I}} \leq n_{\mathrm{D}}=$ $\left|\mathbf{X}_{\mathrm{D}}\right|$ and $|\mathbf{Y} \backslash \mathbf{X}| \leq|\mathbf{X} \backslash \mathbf{Y}|$. Let $\chi^{\prime}: \mathbf{Y}_{\mathrm{I}} \rightarrow \mathbf{X}_{\mathrm{D}}$ be any fixed injection. Then we can obtain an injection $\bar{\chi}: \mathbf{Y} \backslash \mathbf{X} \rightarrow \mathbf{X} \backslash \mathbf{Y}$ such that

$$
\bar{\chi}(\mathbf{y})= \begin{cases}\chi^{-1}(\mathbf{y}) & \text { if } \mathbf{y} \in \mathbf{Y}_{\mathrm{S}} \\ \chi^{\prime}(\mathbf{y}) & \text { if } \mathbf{y} \in \mathbf{Y}_{\mathrm{I}}\end{cases}
$$

Since $|\mathbf{X} \backslash \mathbf{Y}|-|\mathbf{Y} \backslash \mathbf{X}|=\left|\mathbf{X}_{\mathrm{D}}\right|-\left|\mathbf{Y}_{\mathrm{I}}\right|=n_{\mathrm{D}}-n_{\mathrm{I}}$, then by (1),

$$
\begin{aligned}
d_{\bar{\chi}}(\mathbf{Y} \backslash \mathbf{X}, \mathbf{X} \backslash \mathbf{Y})= & \sum_{\mathbf{y} \in \mathbf{Y} \backslash \mathbf{X}} d_{\mathrm{H}}(\mathbf{y}, \bar{\chi}(\mathbf{y}))+L \cdot(|\mathbf{X} \backslash \mathbf{Y}|-|\mathbf{Y} \backslash \mathbf{X}|) \\
= & \sum_{\mathbf{y} \in \mathbf{Y}_{\mathrm{S}}} d_{\mathrm{H}}(\mathbf{y}, \chi(\mathbf{y}))+\sum_{\mathbf{y} \in \mathbf{Y}_{\mathrm{I}}} d_{\mathrm{H}}\left(\mathbf{y}, \chi^{\prime}(\mathbf{y})\right) \\
& +L \cdot\left(n_{\mathrm{D}}-n_{\mathrm{I}}\right) \\
\leq & n_{\mathrm{S}}+L \cdot n_{\mathrm{I}}+L \cdot\left(n_{\mathrm{D}}-n_{\mathrm{I}}\right) \\
= & n_{\mathrm{S}}+L \cdot n_{\mathrm{D}} \\
= & n_{\mathrm{S}}+L \cdot \max \left\{n_{\mathrm{I}}, n_{\mathrm{D}}\right\}
\end{aligned}
$$

where the inequality comes from the simple fact that $d_{\mathrm{H}}\left(\mathbf{z}, \mathbf{z}^{\prime}\right) \leq L$ for any $\mathbf{z}, \mathbf{z}^{\prime} \in \mathbb{A}^{L}$. Hence, by Corollary 1 and Definition 1 , we have

$$
\begin{aligned}
d_{\mathrm{S}}(\mathbf{X}, \mathbf{Y}) & =d_{\mathrm{S}}(\mathbf{X} \backslash \mathbf{Y}, \mathbf{Y} \backslash \mathbf{X}) \\
& \leq d_{\bar{\chi}}(\mathbf{Y} \backslash \mathbf{X}, \mathbf{X} \backslash \mathbf{Y}) \\
& \leq n_{\mathrm{S}}+L \cdot \max \left\{n_{\mathrm{I}}, n_{\mathrm{D}}\right\}
\end{aligned}
$$

Case 2: $n_{\mathrm{I}}>n_{\mathrm{D}}$. In this case, there exists an injection $\chi^{\prime}: \mathbf{X}_{\mathrm{D}} \rightarrow \mathbf{Y}_{\mathrm{I}}$ and we can let $\bar{\chi}: \mathbf{X} \backslash \mathbf{Y} \rightarrow \mathbf{Y} \backslash \mathbf{X}$ be such that

$$
\bar{\chi}(\mathbf{x})= \begin{cases}\chi(\mathbf{x}) & \text { if } \mathbf{x} \in \mathbf{X}_{\mathrm{S}} \\ \chi^{\prime}(\mathbf{x}) & \text { if } \mathbf{x} \in \mathbf{X}_{\mathrm{D}} .\end{cases}
$$

Since $|\mathbf{Y} \backslash \mathbf{X}|-|\mathbf{X} \backslash \mathbf{Y}|=\left|\mathbf{Y}_{\mathrm{I}}\right|-\left|\mathbf{X}_{\mathrm{D}}\right|=n_{\mathrm{I}}-n_{\mathrm{D}}$, then by (1),

$$
\begin{aligned}
d_{\bar{\chi}}(\mathbf{X} \backslash \mathbf{Y}, \mathbf{Y} \backslash \mathbf{X})= & \sum_{\mathbf{x} \in \mathbf{X} \backslash \mathbf{Y}} d_{\mathrm{H}}(\mathbf{x}, \bar{\chi}(\mathbf{x}))+L \cdot(|\mathbf{Y} \backslash \mathbf{X}|-|\mathbf{X} \backslash \mathbf{Y}|) \\
= & \sum_{\mathbf{x} \in \mathbf{X}_{\mathrm{S}}} d_{\mathrm{H}}(\mathbf{x}, \chi(\mathbf{x}))+\sum_{\mathbf{x} \in \mathbf{X}_{\mathrm{D}}} d_{\mathrm{H}}\left(\mathbf{x}, \chi^{\prime}(\mathbf{x})\right) \\
& +L \cdot\left(n_{\mathrm{I}}-n_{\mathrm{D}}\right) \\
\leq & n_{\mathrm{S}}+L \cdot n_{\mathrm{D}}+L \cdot\left(n_{\mathrm{I}}-n_{\mathrm{D}}\right) \\
= & n_{\mathrm{S}}+L \cdot n_{\mathrm{I}} \\
= & n_{\mathrm{S}}+L \cdot \max \left\{n_{\mathrm{I}}, n_{\mathrm{D}}\right\} .
\end{aligned}
$$

Hence, similar to Case 1, we have

$$
\begin{aligned}
d_{\mathrm{S}}(\mathbf{X}, \mathbf{Y}) & =d_{\mathrm{S}}(\mathbf{Y} \backslash \mathbf{X}, \mathbf{X} \backslash \mathbf{Y}) \\
& \leq d_{\bar{\chi}}(\mathbf{X} \backslash \mathbf{Y}, \mathbf{Y} \backslash \mathbf{X}) \\
& \leq n_{\mathrm{S}}+L \cdot \max \left\{n_{\mathrm{I}}, n_{\mathrm{D}}\right\}
\end{aligned}
$$

In both cases, we have $d_{\mathrm{S}}(\mathbf{X}, \mathbf{Y}) \leq n_{\mathrm{S}}+L \cdot \max \left\{n_{\mathrm{I}}, n_{\mathrm{D}}\right\}$, which completes the proof.

Equality in the bound of $d_{\mathrm{S}}(\mathbf{X}, \mathbf{Y})$ in Lemma 3 can be achieved. As an example, consider $\mathbb{A}=\{0,1\}$ and $L=4$, and let the input $\mathbf{X}=\{0011,1010\}$ and output $\mathbf{Y}=$ $\{0111,1010,1100\}$, where 0111 is an erroneous copy of 0011 with one substitution and 1100 is an inserted sequence. Then the error pattern of $\mathbf{Y}$ is $\left(n_{\mathrm{I}}, n_{\mathrm{D}}, n_{\mathrm{S}}\right)=(1,0,1)$ and so $n_{\mathrm{S}}+L \cdot \max \left\{n_{\mathrm{I}}, n_{\mathrm{D}}\right\}$. On the other hand, by (2), we can easily obtain $d_{\mathrm{S}}(\mathbf{X}, \mathbf{Y})=5$. Hence, we have $d_{\mathrm{S}}(\mathbf{X}, \mathbf{Y})=$ $n_{\mathrm{S}}+L \cdot \max \left\{n_{\mathrm{I}}, n_{\mathrm{D}}\right\}$.

For the decoder, when receiving a subset $\mathbf{Y} \subseteq \mathbb{A}^{L}$, its task is to find a possible input subset $\hat{\mathbf{X}} \subseteq \mathbb{A}^{L}$ that is most similar to Y. By the above discussion and Corollary 1 clearly, the sequence-subset distance is a good choice of metric for similarity between $\mathbf{Y}$ and $\hat{\mathbf{X}}$. In the next subsection, we will discuss error-correcting in DNA storage channel using codes with respect to sequence-subset distance. 


\section{Codes with Sequence-Subset Distance}

A sequence-subset code over $\mathbb{A}^{L}$ is a subset $\mathcal{C}$ of the power set $\mathcal{P}\left(\mathbb{A}^{L}\right)$ of the set $\mathbb{A}^{L}$. We call each element of $\mathbb{A}^{L}$ a sequence and call $L$ the sequence length of $\mathcal{C}$. The size $|\mathcal{C}|$ of $\mathcal{C}$ is called the code size of $\mathcal{C}$. In contrast, for each codeword $\mathbf{X} \in \mathcal{C}$, the size of $\mathbf{X}$ (i.e., the number of sequences contained in $\mathbf{X})$ is called the codeword size of $\mathcal{C}$.

Note that a sequence-subset code $\mathcal{C}$ may contain codewords of different sizes. The maximum of codeword sizes of $\mathcal{C}$, i.e., $M=\max \{|\mathbf{X}| ; \mathbf{X} \in \mathcal{C}\}$, is called the maximal codeword size of $\mathcal{C}$. A sequence-subset code $\mathcal{C}$ is said to have constant codeword size (a constant-codeword-size code) if all codewords of $\mathcal{C}$ have the same codeword size. Real DNA storage systems usually use codes with constant codeword size. In fact, using constant-codeword-size codes, the decoder knows how many sequences are stored and hence can easily determine how many sequences are lost during the synthesizing/sequencing process. However, in this work, for the generality of the theory, we allow that different codewords may have different sizes.

The rate of $\mathcal{C}$ is defined as

$$
R(\mathcal{C})=\frac{\log _{q}|\mathcal{C}|}{\log _{q}\left(\sum_{m=0}^{M}\left(\begin{array}{c}
q^{L} \\
m
\end{array}\right)\right)}
$$

and the redundancy of $\mathcal{C}$ is defined as

$$
r(\mathcal{C})=\log _{q}\left(\sum_{m=0}^{M}\left(\begin{array}{l}
q^{L} \\
m
\end{array}\right)\right)-\log _{q}|\mathcal{C}|,
$$

where $q=|\mathbb{A}|$ and $\sum_{m=0}^{M}\left(\begin{array}{c}q^{L} \\ m\end{array}\right)$ is the number of all subsets of $\mathbb{A}^{L}$ of size not greater than $M$ If $\mathcal{C} \subseteq \mathbb{A}^{L}$ is a code with constant codeword size $M$, then the rate and redundancy of $\mathcal{C}$ are defined as

$$
R(\mathcal{C})=\frac{\log _{q}|\mathcal{C}|}{\log _{q}\left(\begin{array}{l}
q^{L} \\
M
\end{array}\right)}
$$

and

$$
r(\mathcal{C})=\log _{q}\left(\begin{array}{l}
q^{L} \\
M
\end{array}\right)-\log _{q}|\mathcal{C}|
$$

respectively, where $\left(\begin{array}{c}q^{L} \\ M\end{array}\right)$ is the number of all subsets of $\mathbb{A}^{L}$ of size $M$.

The minimum distance of a sequence-subset code $\mathcal{C}$, denoted by $d_{\mathrm{S}}(\mathcal{C})$, is the minimum of the sequence-subset distance between any two distinct codewords of $\mathcal{C}$, that is,

$$
d_{\mathbf{S}}(\mathcal{C})=\min \left\{d_{\mathbf{S}}\left(\mathbf{X}, \mathbf{X}^{\prime}\right) ; \mathbf{X}, \mathbf{X}^{\prime} \in \mathcal{C} \text { and } \mathbf{X} \neq \mathbf{X}^{\prime}\right\} .
$$

In general, $L, M,|\mathcal{C}|$ and $d_{\mathrm{S}}(\mathcal{C})$ are four main parameters of $\mathcal{C}$, and we will call $\mathcal{C}$ an $\left(L, M,|\mathcal{C}|, d_{\mathrm{S}}(\mathcal{C})\right)_{q}$ code, where $q$ is the size of the alphabet $\mathbb{A}$.

Suppose $\mathcal{C} \subseteq \mathcal{P}\left(\mathbb{A}^{L}\right)$ is a sequence-subset code. We denote $\overline{\mathcal{C}}=\{\overline{\mathbf{X}} ; \mathbf{X} \in \mathcal{C}\}$, where $\overline{\mathbf{X}}=\mathbb{A}^{L} \backslash \mathbf{X}$. By Corollary 1 for any $\mathbf{X}_{1}, \mathbf{X}_{2} \in \mathcal{C}$, we have $d_{\mathbf{S}}\left(\mathbf{X}_{1}, \mathbf{X}_{2}\right)=d_{\mathbf{S}}\left(\mathbf{X}_{1} \backslash \mathbf{X}_{2}, \mathbf{X}_{2} \backslash \mathbf{X}_{1}\right)=$

${ }^{2}$ In [16], the storage rate of a code $\mathcal{C}$ is defined as $\frac{\log |\mathcal{C}|}{M L}$, where $M$ is the constant codeword size. The definition of the rate $R(\mathcal{C})$ in this work is slightly different from the traditional definition of code rate. This is because for general sequence-subset codes, the size of different codewords may be different. Hence we use $\log _{q}\left(\sum_{m=0}^{M}\left(\begin{array}{c}q^{L} \\ m\end{array}\right)\right)$ rather than $M L$ in the definition of $R(\mathcal{C})$, where $M$ is the maximal codeword size of $\mathcal{C}$.
$d_{\mathrm{S}}\left(\overline{\mathbf{X}_{1}}, \overline{\mathbf{X}_{2}}\right)$. Hence, $\overline{\mathcal{C}}$ and $\mathcal{C}$ have the same sequence length $L$, code size $|\overline{\mathcal{C}}|=|\mathcal{C}|$ and minimum distance $d_{\mathrm{S}}(\mathcal{C})=d_{\mathrm{S}}(\overline{\mathcal{C}})$. For sequence-subset code with constant codeword size $M$, it is assumed that $M \leq \frac{|\mathbb{A}|^{L}}{2}$. Otherwise, we can consider $\overline{\mathcal{C}}$, which has constant codeword size $\bar{M}=|\mathbb{A}|^{L}-M \leq \frac{|\mathbb{A}|^{L}}{2}$.

Aminimum-distance decoder for $\mathcal{C}$ is a function $D$ : $\mathcal{P}\left(\mathbb{A}^{L}\right) \rightarrow \mathcal{C}$ such that for any $\mathbf{Y} \in \mathcal{P}\left(\mathbb{A}^{L}\right)$,

$$
D(\mathbf{Y})=\arg \min _{\mathbf{X}^{\prime} \in \mathcal{C}} d_{\mathbf{S}}\left(\mathbf{X}^{\prime}, \mathbf{Y}\right) .
$$

Theorem 2: Suppose $\mathcal{C}$ has minimum distance $d_{\mathrm{S}}(\mathcal{C})$ and

$$
n_{\mathrm{S}}+L \cdot \max \left\{n_{\mathrm{I}}, n_{\mathrm{D}}\right\} \leq \frac{d_{\mathrm{S}}(\mathcal{C})-1}{2} .
$$

Then any error of pattern $\left(n_{\mathrm{I}}, n_{\mathrm{D}}, n_{\mathrm{S}}\right)$ can be corrected by the minimum-distance decoder for $\mathcal{C}$.

Proof: Let $\mathbf{X}$ be the set of input sequences and $\mathbf{Y}$ be the set of output sequences of the DNA storage channel. By Lemma 3 if $\mathbf{Y}$ has error pattern $\left(n_{\mathrm{I}}, n_{\mathrm{D}}, n_{\mathrm{S}}\right)$, then

$$
d_{\mathrm{S}}(\mathbf{X}, \mathbf{Y}) \leq n_{\mathrm{S}}+L \cdot \max \left\{n_{\mathrm{I}}, n_{\mathrm{D}}\right\} .
$$

Combining this with (3), we have

$$
d_{\mathrm{S}}(\mathbf{X}, \mathbf{Y}) \leq \frac{d_{\mathrm{S}}(\mathcal{C})-1}{2}
$$

Hence, $\mathbf{X}=\arg \min _{\mathbf{X}^{\prime} \in \mathcal{C}} d_{S}\left(\mathbf{X}^{\prime}, \mathbf{Y}\right)=D(\mathbf{Y})$, that is, $\mathbf{X}$ can be correctly recovered by the minimum-distance decoder.

Example 2: Consider $\mathbb{A}=\{0,1\}$ and $L=5$. Let $\mathcal{C}=\left\{\mathbf{X}_{1}, \mathbf{X}_{2}, \mathbf{X}_{3}\right\}$, where $\mathbf{X}_{1}=\{00101,10001\}, \mathbf{X}_{2}=$ $\{01011,10110\}$ and $\mathbf{X}_{3}=\{01000,11100\}$. According to (2), we can obtain $d_{\mathrm{S}}\left(\mathbf{X}_{1}, \mathbf{X}_{2}\right)=d_{\mathrm{S}}\left(\mathbf{X}_{1}, \mathbf{X}_{3}\right)=6$ and $d_{\mathrm{S}}\left(\mathbf{X}_{2}, \mathbf{X}_{3}\right)=4$, hence we have $d_{\mathrm{S}}(\mathcal{C})=4$. By Theorem 2, if $\mathbf{X} \in \mathcal{C}$ is stored (i.e., $\mathbf{X}$ is the input of the DNA storage channel), $\mathbf{Y} \subseteq \mathbb{A}^{5}$ is the read result (i.e., the output of the channel) and the error pattern $\left(n_{\mathrm{I}}, n_{\mathrm{D}}, n_{\mathrm{S}}\right)$ of $\mathbf{Y}$ satisfies $n_{\mathrm{S}}+L \cdot \max \left\{n_{\mathrm{I}}, n_{\mathrm{D}}\right\} \leq \frac{d_{\mathrm{S}}(\mathcal{C})-1}{2}=1$, then the minimum-distance decoder will recover ${ }^{2} \mathbf{X}$ correctly from $\mathbf{Y}$. For example, suppose $\mathbf{X}_{1}$ is stored and the read result is $\mathbf{Y}=\{01101,10001\}$, where 00101 is changed to 01101 by one substitution. We have $d_{\mathrm{S}}\left(\mathbf{X}_{1}, \mathbf{Y}\right)=1$ and $d_{\mathbf{S}}\left(\mathbf{X}_{2}, \mathbf{Y}\right)=$ $d_{\mathrm{S}}\left(\mathbf{X}_{3}, \mathbf{Y}\right)=5$, and then by the minimum-distance decoder, $D(\mathbf{Y})=\arg \min _{\mathbf{X}^{\prime} \in \mathcal{C}} d_{\mathbf{S}}\left(\mathbf{X}^{\prime}, \mathbf{Y}\right)=\mathbf{X}_{1}$.

Similar to the classical error-correcting codes, the inequality (3) is a sufficient condition for an output with error pattern $\left(n_{\mathrm{I}}, n_{\mathrm{D}}, n_{\mathrm{S}}\right)$ to be corrected by the minimum-distance decoder but not a necessary condition. To illustrate this, reconsider Example 2. Now, suppose $\mathbf{X}_{1}$ is stored and the read result is $\mathbf{Y}=\{01101\}$, where 10001 is lost and 00101 is changed to 01101 by one substitution. In this case, we have $\left(n_{\mathrm{I}}, n_{\mathrm{D}}, n_{\mathrm{S}}\right)=$ $(0,1,1)$, and so $n_{\mathrm{S}}+L \cdot \max \left\{n_{\mathrm{I}}, n_{\mathrm{D}}\right\}=6>\frac{3}{2}=\frac{d_{\mathrm{S}}(\mathcal{C})-1}{2}$. However, $\mathbf{X}_{1}$ can be correctly recovered from $Y$ by the minimum-distance decoder because $d_{\mathrm{S}}\left(\mathbf{X}_{1}, \mathbf{Y}\right)=6<7=$ $d_{\mathrm{S}}\left(\mathbf{X}_{2}, \mathbf{Y}\right)=d_{\mathrm{S}}\left(\mathbf{X}_{3}, \mathbf{Y}\right)$.

Remark 2: Suppose the channel input is $\mathbf{X}$ and output is $\mathbf{Y}$ such that the error pattern of $\mathbf{Y}$ is $\left(n_{\mathrm{I}}, n_{\mathrm{D}}, n_{\mathrm{S}}\right)$. It is sufficient to assume that $n_{\mathrm{I}}=0$ or $n_{\mathrm{D}}=0$. In fact, suppose $n_{\mathrm{I}} \geq n_{\mathrm{D}}$. Denoted by $\mathbf{x}_{1}, \cdots, \mathbf{x}_{n_{\mathrm{D}}}$ the $n_{\mathrm{D}}$ deleted sequences and $\mathbf{y}_{1}, \cdots, \mathbf{y}_{n_{\mathrm{I}}}$ the $n_{\mathrm{I}}$ inserted sequences. For each $i \in\left[n_{\mathrm{D}}\right]$, 
we view $\mathbf{y}_{i}$ as obtained from $\mathbf{x}_{i}$ by symbol substitutions. Then the error pattern is $\left(n_{\mathrm{I}}^{\prime}, n_{\mathrm{D}}^{\prime}, n_{\mathrm{S}}^{\prime}\right)=\left(n_{\mathrm{I}}-n_{\mathrm{D}}, 0, n_{\mathrm{S}}+\overline{n_{\mathrm{S}}}\right)$, where $\overline{n_{\mathrm{S}}}$ is the total substitutions in $\mathbf{y}_{1}, \cdots, \mathbf{y}_{n_{\mathrm{D}}}$. Noticing that the number of substitutions in each sequence is not greater than its length $L$, then the total substitutions $\overline{n_{\mathrm{S}}} \leq n_{\mathrm{D}} L$, and hence $n_{\mathrm{S}}^{\prime}+L \max \left\{n_{\mathrm{I}}^{\prime}, n_{\mathrm{D}}^{\prime}\right\}=n_{\mathrm{S}}+\overline{n_{\mathrm{S}}}+\left(n_{\mathrm{I}}-n_{\mathrm{D}}\right) L \leq$ $n_{\mathrm{S}}+n_{\mathrm{D}} L+\left(n_{\mathrm{I}}-n_{\mathrm{D}}\right) L=n_{\mathrm{S}}+n_{\mathrm{I}} L=n_{\mathrm{S}}+L \max \left\{n_{\mathrm{I}}, n_{\mathrm{D}}\right\}$. By Theorem 2] if $\mathbf{Y}$ with error pattern $\left(n_{\mathrm{I}}, n_{\mathrm{D}}, n_{\mathrm{S}}\right)$ can be corrected, then $\mathbf{Y}$ with error pattern $\left(n_{\mathrm{I}}^{\prime}, n_{\mathrm{D}}^{\prime}, n_{\mathrm{S}}^{\prime}\right)$ can also be corrected. Similarly, if $n_{\mathrm{I}}<n_{\mathrm{D}}$, then by viewing each $\mathbf{y}_{i}$, $i \in\left[n_{\mathrm{I}}\right]$, as obtained from $\mathbf{x}_{i}$ by symbol substitutions, we can obtain an error pattern $\left(n_{\mathrm{I}}^{\prime}, n_{\mathrm{D}}^{\prime}, n_{\mathrm{S}}^{\prime}\right)=\left(0, n_{\mathrm{D}}-n_{\mathrm{I}}, n_{\mathrm{S}}+\overline{n_{\mathrm{S}}}\right)$ such that if $\mathbf{Y}$ with error pattern $\left(n_{\mathrm{I}}, n_{\mathrm{D}}, n_{\mathrm{S}}\right)$ can be corrected, then $\mathbf{Y}$ with error pattern $\left(n_{\mathrm{I}}^{\prime}, n_{\mathrm{D}}^{\prime}, n_{\mathrm{S}}^{\prime}\right)$ can also be corrected. Thus, we can always assume that $n_{\mathrm{I}}=0$ or $n_{\mathrm{D}}=0$.

In [14] and [15], it was assumed that the number of output sequences is not greater than the number of input sequences. In this work, considering the sequence insertion errors, we allow that the number of output sequences of the DNA storage channel can be larger than the number of input sequences. This assumption is also of interest for the generality of the theory.

Usually, correction of sequence insertion/deletion requires codes with larger minimum distance. To see this, consider two special cases of error pattern. The first special case is $n_{\mathrm{I}}=n_{\mathrm{D}}=0$. In this case, by Theorem 2 , a sequence-subset code $\mathcal{C}$ with minimum distance $d_{\mathrm{S}}(\mathcal{C})$ can correct a total of at most $\frac{d_{\mathrm{S}}(\mathcal{C})-1}{2}$ substitution errors. The second special case is $n_{\mathrm{I}}=n_{\mathrm{S}}=0$. A sequence-subset code $\mathcal{C}$ with minimum distance $d_{\mathrm{S}}(\mathcal{C})$ can correct a total of at most $\frac{d_{\mathrm{S}}(\mathcal{C})-1}{2 L}$ sequencedeletions, which is $\frac{1}{L}$ of the number of correctable substitution errors.

\section{Bounds on the Size of Sequence Subset Codes}

In this Section, we consider codes with constant codeword size. Let $S_{q}(L, M, d)$ denote the maximum number of codewords in a sequence-subset code over a $q$-ary alphabet with sequence length $L$, constant codeword size $M$ and minimum sequence-subset distance at least $d$. A $q$-ary sequence-subset code is said to be optimal (with respect to code size) if it has the largest possible code size of any $q$-ary sequence-subset code of the given parameters $L, M$ and $d$. In this section, we always assume that $\mathbb{A}$ is an alphabet of size $q$. We will derive some upper bounds on $S_{q}(L, M, d)$.

Clearly, for any sequence-subset code $\mathcal{C} \subseteq \mathcal{P}\left(\mathbb{A}^{L}\right)$ with constant codeword size $M$, its minimum distance $d_{\mathrm{S}}(\mathcal{C}) \leq$ $L M$, and hence $M \geq \frac{d_{\mathrm{s}}(\mathcal{C})}{L}$. For this reason, in the following, we always assume that $d \leq L M$, or equivalently, $M \geq\left\lceil\frac{d}{L}\right\rceil$.

\section{A. Upper Bound for the Special Case $d=L M$}

First, consider the special case that $d=L M$. We have the following upper bound on $S_{q}(L, M, d)$.

Theorem 3: Suppose $d=L M$. Then

$$
S_{q}(L, M, d) \leq\left\lfloor q M^{-\frac{1}{L}}\right\rfloor
$$

Proof: Let $\mathcal{C}=\left\{\mathbf{X}_{1}, \mathbf{X}_{2}, \cdots, \mathbf{X}_{N}\right\} \subseteq \mathcal{P}\left(\mathbb{A}^{L}\right)$ be an arbitrary sequence-subset code with constant codeword size
$M$ and minimum distance $d$, where for each $i \in[N], \mathbf{X}_{i}=$ $\left\{\mathbf{x}_{i, 1}, \mathbf{x}_{i, 2}, \cdots, \mathbf{x}_{i, M}\right\} \subseteq \mathbb{A}^{L}$. We will prove that $N \leq q M^{-\frac{1}{L}}$. For each fixed $\ell \in[L]$ and $i \in[N]$, let

$$
W_{i, \ell}=\bigcup_{j \in[M]}\left\{\mathbf{x}_{i, j}(\ell)\right\}
$$

Then we have the following Claim.

Claim 1: For each fixed $\ell \in[L]$ and $i \in[N]$, $W_{1, \ell}, W_{2, \ell}, \cdots, W_{N, \ell}$ are mutually disjoint subsets of $\mathbb{A}$.

To prove Claim 1, we first notice that for any distinct $i_{1}, i_{2} \in$ $[N]$ and any (not necessarily distinct) $j_{1}, j_{2} \in[M]$,

$$
d_{\mathrm{H}}\left(\mathbf{x}_{i_{1}, j_{1}}, \mathbf{x}_{i_{2}, j_{2}}\right)=L,
$$

which can be proved as follows. Since both $\mathbf{x}_{i_{1}, j_{1}}$ and $\mathbf{x}_{i_{2}, j_{2}}$ have length $L$, we have $d_{\mathrm{H}}\left(\mathbf{x}_{i_{1}, j_{1}}, \mathbf{x}_{i_{2}, j_{2}}\right) \leq L$. We can only have $d_{\mathrm{H}}\left(\mathbf{x}_{i_{1}, j_{1}}, \mathbf{x}_{i_{2}, j_{2}}\right)=L$ because otherwise, we have $d_{\mathrm{H}}\left(\mathbf{x}_{i_{1}, j_{1}}, \mathbf{x}_{i_{2}, j_{2}}\right)<L$ and we can construct a bijection $\chi: \mathbf{X}_{i_{1}} \rightarrow \mathbf{X}_{i_{2}}$ such that $\chi\left(\mathbf{x}_{i_{1}, j_{1}}\right)=\mathbf{x}_{i_{2}, j_{2}}$. Since for all $\mathbf{x}_{i_{1}, j} \in \mathbf{X}_{i_{1}}$ and $\mathbf{x}_{i_{1}, j^{\prime}} \in \mathbf{X}_{i_{2}}, d_{\mathrm{H}}\left(\mathbf{x}_{i_{1}, j}, \mathbf{x}_{i_{1}, j^{\prime}}\right) \leq L$ (the length of $\mathbf{x}_{i_{1}, j}$ and $\mathbf{x}_{i_{2}, j^{\prime}}$ ), then by (1) we can obtain $d_{\chi}\left(\mathbf{X}_{i_{1}}, \mathbf{X}_{i_{2}}\right)<L M$, and further by Definition 11 we have $d_{\mathrm{S}}\left(\mathbf{X}_{i_{1}}, \mathbf{X}_{i_{2}}\right)<L M$, which contradicts to the assumption that the minimum distance of $\mathcal{C}$ is $d=L M$. Hence, (5) must hold. Again since both $\mathbf{x}_{i_{1}, j_{1}}$ and $\mathbf{x}_{i_{2}, j_{2}}$ have length $L$, then (5) implies that for any fixed $\ell \in[L], \mathbf{x}_{i_{1}, j_{1}}(\ell) \neq \mathbf{x}_{i_{2}, j_{2}}(\ell)$. Since $j_{1}, j_{2}$ are any elements of $[M]$, then we have $W_{i_{1}, \ell} \cap W_{i_{2}, \ell}=\emptyset$. Further, since $i_{1}, i_{2}$ are any distinct elements of $[N]$, we have $W_{1, \ell}, W_{2, \ell}, \cdots, W_{N, \ell}$ are mutually disjoint subsets of $\mathbb{A}$, which proves Claim 1 .

Now, by Claim 1, we have

$$
\sum_{i=1}^{N}\left|W_{i, \ell}\right| \leq|\mathbb{A}|=q
$$

By the construction of $W_{i, \ell}$, for each $i \in[N]$ and $j \in[M]$, we have $\mathbf{x}_{i, j} \in W_{i, 1} \times W_{i, 2} \times \cdots \times W_{i, L}$, which implies that $\mathbf{X}_{i}=\left\{\mathbf{x}_{i, 1}, \mathbf{x}_{i, 2}, \cdots, \mathbf{x}_{i, M}\right\} \subseteq W_{i, 1} \times W_{i, 2} \times \cdots \times W_{i, L}$, and hence we have

$$
\left|W_{i, 1} \times W_{i, 2} \times \cdots \times W_{i, L}\right|=\prod_{\ell=1}^{L}\left|W_{i, \ell}\right| \geq\left|\mathbf{X}_{i}\right|=M .
$$

Now, consider (6). By the inequality of arithmetic and geometric means, for each $\ell \in[L]$, we have

$$
\frac{q}{N} \geq \frac{1}{N} \sum_{i=1}^{N}\left|W_{i, \ell}\right| \geq\left(\prod_{i=1}^{N}\left|W_{i, \ell}\right|\right)^{\frac{1}{N}}
$$

Combining this with (7), we have

$$
\begin{aligned}
\left(\frac{q}{N}\right)^{L} & \geq \prod_{\ell=1}^{L}\left(\prod_{i=1}^{N}\left|W_{i, \ell}\right|\right)^{\frac{1}{N}} \\
& =\prod_{i=1}^{N}\left(\prod_{\ell=1}^{L}\left|W_{i, \ell}\right|\right)^{\frac{1}{N}} \\
& \geq\left(M^{\frac{1}{N}}\right)^{N} \\
& =M .
\end{aligned}
$$


From this we have $\frac{q}{N} \geq M^{\frac{1}{L}}$, which implies $N \leq q M^{-\frac{1}{L}}$. principle [24], we have

Hence,

$$
S_{q}(L, M, d) \leq q M^{-\frac{1}{L}}
$$

Since $S_{q}(L, M, d)$ is an integer, so

$$
S_{q}(L, M, d) \leq\left\lfloor q M^{-\frac{1}{L}}\right\rfloor
$$

which completes the proof.

Consider the redundancy of the codes. For any code $\mathcal{C} \subseteq$ $\mathbb{A}^{L}$ with constant codeword size $M$ and minimum distance $d=L M$, by Theorem 3 , we have

$$
\begin{aligned}
r(\mathcal{C}) & =\log _{q}\left(\begin{array}{c}
q^{L} \\
M
\end{array}\right)-\log _{q}|\mathcal{C}| \\
& \geq \log _{q}\left(\begin{array}{c}
q^{L} \\
M
\end{array}\right)-\log _{q}\left(q M^{-\frac{1}{L}}\right) \\
& =\log _{q} \frac{q^{L} ! M^{\frac{1}{L}}}{M !\left(q^{L}-M\right) ! q}
\end{aligned}
$$

The bound (4) is tight for the special case that $M^{\frac{1}{L}}$ is an integer. Codes that achieve equality of (4) are constructed in Section IV.A.

\section{B. Plotkin-like Bound}

We present the Plotkin-like Bound of sequence-subset codes as the following theorem.

Theorem 4 (Plotkin-like Bound): Let $\mathcal{C}$ be an $(L, M, N, d)_{q}$ code such that $r L M<d$, where $r=1-\frac{1}{q}$. Then

$$
N \leq \frac{d}{d-r L M}
$$

Proof: Our proof of this theorem is similar to the proof of [23, Theorem 2.2.1].

Suppose $\mathcal{C}=\left\{\mathbf{X}_{1}, \mathbf{X}_{2}, \cdots, \mathbf{X}_{N}\right\}$ such that for each $i \in$ $[N], \mathbf{X}_{i}=\left\{\mathbf{x}_{i, 1}, \mathbf{x}_{i, 2}, \cdots, \mathbf{x}_{i, M}\right\} \subseteq \mathbb{A}^{L}$. First, we have the following claim, which we will prove later.

Claim 2: For any distinct $i_{1}, i_{2} \in[N]$, we have

$$
d_{\mathrm{S}}\left(\mathbf{X}_{i_{1}}, \mathbf{X}_{i_{2}}\right) \leq \frac{1}{M} \sum_{j_{1}, j_{2} \in[M]} d_{\mathrm{H}}\left(\mathbf{x}_{i_{1}, j_{1}}, \mathbf{x}_{i_{2}, j_{2}}\right) .
$$

Now, let

$$
A=\sum_{i_{1}, i_{2} \in[N]} \sum_{j_{1}, j_{2} \in[M]} d_{\mathrm{H}}\left(\mathbf{x}_{i_{1}, j_{1}}, \mathbf{x}_{i_{2}, j_{2}}\right) .
$$

Since $d$ is the minimum distance of $\mathcal{C}$, by the averaging

$$
\begin{aligned}
d & \leq\left(\begin{array}{c}
N \\
2
\end{array}\right)^{-1} \sum_{\left\{i_{1}, i_{2}\right\} \subseteq[N]} d_{\mathrm{S}}\left(\mathbf{X}_{i_{1}}, \mathbf{X}_{i_{2}}\right) \\
& =\frac{1}{2}\left(\begin{array}{c}
N \\
2
\end{array}\right)^{-1} \sum_{i_{1}, i_{2} \in[N], i_{1} \neq i_{2}} d_{\mathrm{S}}\left(\mathbf{X}_{i_{1}}, \mathbf{X}_{i_{2}}\right) \\
& \leq \frac{1}{2}\left(\begin{array}{c}
N \\
2
\end{array}\right)^{-1} \sum_{i_{1}, i_{2} \in[N]} d_{\mathrm{S}}\left(\mathbf{X}_{i_{1}}, \mathbf{X}_{i_{2}}\right) \\
& \leq \frac{1}{N(N-1)} \sum_{i_{1}, i_{2} \in[N]}\left(\frac{1}{M} \sum_{j_{1}, j_{2} \in[M]} d_{\mathrm{H}}\left(\mathbf{x}_{i_{1}, j_{1}}, \mathbf{x}_{i_{2}, j_{2}}\right)\right) \\
& =\frac{1}{N(N-1)} \frac{1}{M} \cdot A
\end{aligned}
$$

where the last inequality is obtained by Claim 2 .

For each $a \in \mathbb{A}$ and $\ell \in[L]$, let $n_{\ell, a}$ be the number of $(i, j) \in[N] \times[M]$ such that $\mathbf{x}_{i, j}(\ell)=a$. Then for each fixed $\ell \in[L]$, we have

$$
\sum_{a \in \mathbb{A}} n_{\ell, a}=N M
$$

Moreover, we have

$$
\begin{aligned}
A & =\sum_{i_{1}, i_{2} \in[N]} \sum_{j_{1}, j_{2} \in[M]} d_{\mathrm{H}}\left(\mathbf{x}_{i_{1}, j_{1}}, \mathbf{x}_{i_{2}, j_{2}}\right) \\
& =\sum_{\ell=1}^{L} \sum_{a \in \mathbb{A}} n_{\ell, a}\left(N M-n_{\ell, a}\right) \\
& =L(N M)^{2}-\sum_{\ell=1}^{L} \sum_{a \in \mathbb{A}} n_{\ell, a}^{2} .
\end{aligned}
$$

For each $\ell \in[L]$, by the Cauchy-Schwartz inequality,

$$
\left(\sum_{a \in \mathbb{A}} n_{\ell, a}\right)^{2} \leq q \sum_{a \in \mathbb{A}} n_{\ell, a}^{2}
$$

where $q=|\mathbb{A}|$. Combining this with $[10$, we obtain

$$
\begin{aligned}
A & \leq L(N M)^{2}-\sum_{\ell=1}^{L} \frac{1}{q}\left(\sum_{a \in \mathbb{A}} n_{\ell, a}\right)^{2} \\
& =L(N M)^{2}-\sum_{\ell=1}^{L} \frac{1}{q}(N M)^{2} \\
& =\left(1-\frac{1}{q}\right) L(N M)^{2},
\end{aligned}
$$

where the first equality is obtained from (9). Combining 8 and (11), we obtain

$$
d \leq \frac{1}{N(N-1)} \frac{1}{M} \cdot\left(1-\frac{1}{q}\right) L(N M)^{2} .
$$

Solving $N$ from the above inequality we obtain

$$
N \leq \frac{d}{d-r L M}
$$

where $r=1-\frac{1}{q}$.

To complete the proof of Theorem 4 we still need to prove Claim 2. 
Proof of Claim 2: Let $\mathscr{S}_{M}$ denote the permutation group on $[M]$. Note that for any $j_{1}, j_{2} \in[M]$, not necessarily distinct, there are $(M-1)$ ! permutations $\chi \in \mathscr{S}_{M}$ such that $\chi\left(j_{1}\right)=j_{2}$. We have

$$
\begin{aligned}
& \sum_{\chi \in \mathscr{S}_{M}} \sum_{j \in[M]} d_{\mathrm{H}}\left(\mathbf{x}_{i_{1}, j}, \mathbf{x}_{i_{2}, \chi(j)}\right) \\
& =(M-1) ! \sum_{j_{1}, j_{2} \in[M]} d_{\mathrm{H}}\left(\mathbf{x}_{i_{1}, j_{1}}, \mathbf{x}_{i_{2}, j_{2}}\right) .
\end{aligned}
$$

Further, by Definition 1 and the averaging principle [24], we have

$$
\begin{aligned}
d_{\mathrm{S}}\left(\mathbf{X}_{i_{1}}, \mathbf{x}_{i_{2}}\right) & \leq \frac{1}{M !} \sum_{\chi \in \mathscr{S}_{M}} d_{\chi}\left(\mathbf{x}_{i_{1}}, \mathbf{x}_{i_{2}}\right) \\
& =\frac{1}{M !} \sum_{\chi \in \mathscr{S}_{M}} \sum_{j \in[M]} d_{\mathrm{H}}\left(\mathbf{x}_{i_{1}, j}, \mathbf{x}_{i_{2}, \chi(j)}\right) \\
& =\frac{(M-1) !}{M !} \sum_{j_{1}, j_{2} \in[M]} d_{\mathrm{H}}\left(\mathbf{x}_{i_{1}, j_{1}}, \mathbf{x}_{i_{2}, j_{2}}\right) \\
& =\frac{1}{M} \sum_{j_{1}, j_{2} \in[M]} d_{\mathrm{H}}\left(\mathbf{x}_{i_{1}, j_{1}}, \mathbf{x}_{i_{2}, j_{2}}\right),
\end{aligned}
$$

where the second equality comes from (12).

Consider the redundancy of the codes. For any code $\mathcal{C} \subseteq$ $\mathbb{A}^{L}$ with constant codeword size $M$ and minimum distance $d>r L M$, where $r=1-\frac{1}{q}$, by Theorem 4, we have

$$
\begin{aligned}
r(\mathcal{C}) & =\log _{q}\left(\begin{array}{l}
q^{L} \\
M
\end{array}\right)-\log _{q}|\mathcal{C}| \\
& \geq \log _{q}\left(\begin{array}{c}
q^{L} \\
M
\end{array}\right)-\log _{q}\left(\frac{d}{d-r L M}\right) \\
& =\log _{q} \frac{q^{L} !(d-r L M)}{M !\left(q^{L}-M\right) ! d} .
\end{aligned}
$$

For the special case that $d=L M$, we have $\frac{d}{d-r L M}=q>$ $q M^{-\frac{1}{L}}$, where $r=1-\frac{1}{q}$. Thus, the bound given in Theorem 3 is tighter than the bound given in Theorem 4 for $M>1$. It is still not known whether the bound in Theorem 4 is achievable for other cases.

\section{Singleton-like Bound}

For each code $\mathcal{C}=\left\{\mathbf{X}_{1}, \mathbf{X}_{2}, \cdots, \mathbf{X}_{N}\right\} \subseteq \mathcal{P}\left(\mathbb{A}^{L}\right)$, denote

$$
V(\mathcal{C})=\bigcup_{i=1}^{N} \mathbf{X}_{i}
$$

Further, let $\tilde{S}_{q}(L, M, K, d)$ denote the maximum number of codewords in a sequence-subset code $\mathcal{C}$ over a $q$-ary alphabet $\mathbb{A}$ with sequence length $L$, constant codeword size $M$, minimum sequence-subset distance at least $d$ and $|V(\mathcal{C})| \leq K$. Clearly, for any $K \leq q^{L}$,

$$
\tilde{S}_{q}(L, M, K, d) \leq \tilde{S}_{q}\left(L, M, q^{L}, d\right)=S_{q}(L, M, d) .
$$

We first prove a recursive bound on $\tilde{S}_{q}(L, M, K, d)$ in the following theorem.
Theorem 5: Suppose $d \leq L M$ and $K \leq q^{L}$. We have

$$
\tilde{S}_{q}(L, M, K, d) \leq\left\lfloor\frac{K}{M} \tilde{S}_{q}(L, M-1, K-1, d)\right\rfloor .
$$

Proof: Let $\mathcal{C}=\left\{\mathbf{X}_{1}, \mathbf{X}_{2}, \cdots, \mathbf{X}_{N}\right\} \subseteq \mathcal{P}\left(\mathbb{A}^{L}\right)$ be a sequence-subset code with constant codeword size $M$, minimum distance at least $d$ such that $|V(\mathcal{C})| \leq K$ and code size $|\mathcal{C}|=N=\tilde{S}_{q}(L, M, K, d)$, where $\mathbf{X}_{i} \subseteq \mathbb{A}^{\bar{L}}$ for each $i \in[N]$.

For each $\mathbf{x} \in V(\mathcal{C})$, let

$$
\mathcal{C}(\mathbf{x})=\{\mathbf{X} \in \mathcal{C} ; \mathbf{x} \in \mathbf{X}\}
$$

and

$$
\tilde{\mathcal{C}}(\mathbf{x})=\{\tilde{\mathbf{X}}=\mathbf{X} \backslash\{\mathbf{x}\} ; \mathbf{X} \in \mathcal{C}(\mathbf{x})\} .
$$

Then $\tilde{\mathcal{C}}(\mathbf{x}) \subseteq \mathcal{P}\left(\mathbb{A}^{L}\right)$ has constant codeword size $M-1$, size $|\tilde{\mathcal{C}}(\mathbf{x})|=|\mathcal{C}(\mathbf{x})|$ and $|V(\tilde{\mathcal{C}}(\mathbf{x}))| \leq K-1$.

Moreover, for any distinct $\tilde{\mathbf{X}}_{i_{1}}, \tilde{\mathbf{X}}_{i_{2}} \in \tilde{\mathcal{C}}(\mathbf{x})$, by the construction of $\tilde{\mathcal{C}}(\mathbf{x})$, we have $\tilde{\mathbf{X}}_{i_{1}}=\mathbf{X}_{i_{1}} \backslash\{\mathbf{x}\}$ and $\tilde{\mathbf{X}}_{i_{2}}=\mathbf{X}_{i_{2}} \backslash\{\mathbf{x}\}$ for some distinct $\mathbf{X}_{i_{1}}, \mathbf{X}_{i_{2}} \in \mathcal{C}(\mathbf{x})$, so $\tilde{\mathbf{X}}_{i_{1}} \backslash \tilde{\mathbf{X}}_{i_{2}}=\mathbf{X}_{i_{1}} \backslash \mathbf{X}_{i_{2}}$ and $\tilde{\mathbf{X}}_{i_{2}} \backslash \tilde{\mathbf{X}}_{i_{1}}=\mathbf{X}_{i_{2}} \backslash \mathbf{X}_{i_{1}}$. By Corollary 1, we have

$$
d_{\mathrm{S}}\left(\tilde{\mathbf{X}}_{i_{1}}, \tilde{\mathbf{X}}_{i_{2}}\right)=d_{\mathrm{S}}\left(\mathbf{X}_{i_{1}}, \mathbf{X}_{i_{2}}\right) .
$$

Then we have $d_{\mathrm{S}}(\tilde{\mathcal{C}}(\mathbf{x}))=d_{\mathrm{S}}(\mathcal{C}(\mathbf{x}))$. On the other hand, since $\mathcal{C}(\mathbf{x}) \subseteq \mathcal{C}$, we have $d_{\mathrm{S}}(\mathcal{C}(\mathbf{x})) \geq d_{\mathrm{S}}(\mathcal{C}) \geq d$. Thus, $d_{\mathrm{S}}(\tilde{\mathcal{C}}(\mathbf{x})) \geq$ d.

By the above discussion, for each $\mathbf{x} \in V(\mathcal{C})$, we have

$$
|\tilde{\mathcal{C}}(\mathbf{x})| \leq \tilde{S}_{q}(L, M-1, K-1, d) .
$$

Now, we estimate $|\tilde{\mathcal{C}}(\mathbf{x})|$. Since $|\tilde{\mathcal{C}}(\mathbf{x})|=|\mathcal{C}(\mathbf{x})|$, it is sufficient to estimate $|\mathcal{C}(\mathbf{x})|$. Denote $V(\mathcal{C})=\left\{\mathbf{x}_{1}, \mathbf{x}_{2}, \cdots, \mathbf{x}_{\tilde{K}}\right\}$, where $\tilde{K}=|V(\mathcal{C})|$. Consider the $N \times \tilde{K}$ matrix $I=\left(a_{i, j}\right)$ such that $a_{i, j}=1$ if $\mathbf{x}_{j} \in \mathbf{X}_{i}$, and $a_{i, j}=0$ otherwise. Note that the number of ones in row $i$ of $I$ is $\left|\mathbf{X}_{i}\right|=M$ and the number of ones in column $j$ of $I$ is $\left|\mathcal{C}\left(\mathbf{x}_{j}\right)\right|$. By counting the total number of ones in $I$, we obtain

$$
\sum_{\mathbf{x} \in V(\mathcal{C})}|\mathcal{C}(\mathbf{x})|=\sum_{\mathbf{x} \in \mathcal{C}}|\mathbf{X}|=M N
$$

By the averaging principle [24], there exists an $\mathbf{x}_{j_{0}} \in V(\mathcal{C})$ such that

$$
\left|\mathcal{C}\left(\mathbf{x}_{j_{0}}\right)\right| \geq \frac{M N}{|V(\mathcal{C})|} \geq \frac{M N}{K} .
$$

Hence,

$$
N \leq \frac{K}{M}\left|\mathcal{C}\left(\mathbf{x}_{j_{0}}\right)\right|=\frac{K}{M}\left|\tilde{\mathcal{C}}\left(\mathbf{x}_{j_{0}}\right)\right| .
$$

Note that $|\mathcal{C}|=\tilde{S}_{q}(L, M, K, d)=N$. Then we have

$$
\tilde{S}_{q}(L, M, K, d) \leq \frac{K}{M}\left|\tilde{\mathcal{C}}\left(\mathbf{x}_{0}\right)\right| .
$$

This, combining with (16), implies that

$$
\tilde{S}_{q}(L, M, K, d) \leq \frac{K}{M} \tilde{S}_{q}(L, M-1, K-1, d) .
$$

Noticing that $\tilde{S}_{q}(L, M, K, d)$ is an integer, then

$$
\tilde{S}_{q}(L, M, K, d) \leq\left\lfloor\frac{K}{M} \tilde{S}_{q}(L, M-1, K-1, d)\right\rfloor,
$$

which completes the proof. 
Now, we can prove a Singleton-like bound for sequencesubset codes as follows.

Theorem 6 (Singleton-like Bound): Suppose $r L M_{0}<d \leq$ $L M_{0}$, where $r=1-\frac{1}{q}$ and $M_{0}=\left\lceil\frac{d}{L}\right\rceil$. Then

$S_{q}(L, M, d)$

$\leq\left\lfloor\frac{q^{L}}{M}\left\lfloor\frac{q^{L}-1}{M-1} \cdots\left\lfloor\frac{q^{L}-M+M_{0}+1}{M_{0}+1} f\left(L, M_{0}, d\right)\right\rfloor \ldots\right\rfloor\right\rfloor$,

where

$$
f\left(L, M_{0}, d\right)= \begin{cases}\left\lfloor q M_{0}^{-\frac{1}{L}}\right\rfloor & \text { if } d=L M_{0} ; \\ \frac{d}{d-r L M_{0}} & \text { if } r L M_{0}<d<L M_{0} .\end{cases}
$$

Proof: Repeatedly using Theorem 5 with $M-M_{0}$ times, we obtain

$$
\begin{aligned}
\tilde{S}_{q}\left(L, M, q^{L}, d\right) \leq & \left\lfloor\frac { q ^ { L } } { M } \left\lfloor\frac { q ^ { L } - 1 } { M - 1 } \cdots \left\lfloor\frac{q^{L}-M+M_{0}+1}{M_{0}+1}\right.\right.\right. \\
& \left.\left.\left.\tilde{S}_{q}\left(L, M_{0}, q^{L}-M+M_{0}, d\right)\right\rfloor \ldots\right\rfloor\right\rfloor .
\end{aligned}
$$

Moreover, according to 14, we have

$$
S_{q}(L, M, d)=\tilde{S}_{q}\left(L, M, q^{L}, d\right)
$$

and

$$
\begin{aligned}
\tilde{S}_{q}\left(L, M_{0}, q^{L}-M+M_{0}, d\right) & \leq S_{q}\left(L, M_{0}, q^{L}, d\right) \\
& =S_{q}\left(L, M_{0}, d\right) .
\end{aligned}
$$

Combining the above three equations, we have

$$
\begin{aligned}
S_{q}(L, M, d) \leq & \left\lfloor\frac{q^{L}}{M}\left\lfloor\frac{q^{L}-1}{M-1} \cdots\right\rfloor \frac{q^{L}-M+M_{0}+1}{M_{0}+1}\right. \\
& \left.\left.\left.S_{q}\left(L, M_{0}, d\right)\right\rfloor \cdots\right\rfloor\right\rfloor .
\end{aligned}
$$

Let $f\left(L, M_{0}, d\right)$ be defined as in 177. By Theorem 3 and Theorem 4, we have

$$
S_{q}\left(L, M_{0}, d\right) \leq f\left(L, M_{0}, d\right) .
$$

Combining this with 18, we have

$$
\begin{aligned}
S_{q}(L, M, d) \leq & \left\lfloor\frac{q^{L}}{M}\left\lfloor\frac{q^{L}-1}{M-1} \cdots\right\rfloor \frac{q^{L}-M+M_{0}+1}{M_{0}+1}\right. \\
& \left.\left.f\left(L, M_{0}, d\right)\right\rfloor \cdots\right\rfloor,
\end{aligned}
$$

which completes the proof.

Remark 3: It is easy to see that

$$
\begin{aligned}
& \left\lfloor\frac{q^{L}}{M}\left\lfloor\frac{q^{L}-1}{M-1} \cdots\left\lfloor\frac{q^{L}-M+M_{0}+1}{M_{0}+1} f\left(L, M_{0}, d\right)\right\rfloor \ldots\right\rfloor\right\rfloor \\
& \leq\left(\prod_{k=0}^{M-M_{0}-1} \frac{q^{L}-k}{M-k}\right) f\left(L, M_{0}, d\right)
\end{aligned}
$$

and

$$
\left(\begin{array}{l}
q^{L} \\
M
\end{array}\right)=\left(\prod_{k=0}^{M-M_{0}-1} \frac{q^{L}-k}{M-k}\right)\left(\begin{array}{c}
q^{L}-M+M_{0} \\
M_{0}
\end{array}\right) .
$$

Hence, the bound in Theorem 6 gives a bound on the code rate as

$$
\frac{S_{q}(L, M, d)}{\left(\begin{array}{l}
q^{L} \\
M
\end{array}\right)} \leq \frac{1}{\left(\begin{array}{c}
q^{L}-M+M_{0} \\
M_{0}
\end{array}\right)} \cdot f\left(L, M_{0}, d\right),
$$

where $f\left(L, M_{0}, d\right)$ is defined as in 17 .

Consider the redundancy of the codes. For any code $\mathcal{C} \subseteq$ $\mathbb{A}^{L}$ with constant codeword size $M$ and minimum distance $d$ satisfying $r L M_{0}<d \leq L M_{0}$, where $r=1-\frac{1}{q}$ and $M_{0}=$ $\left\lceil\frac{d}{L}\right\rceil$, by Remark 3] we have

$$
S_{q}(L, M, d) \leq\left(\begin{array}{c}
q^{L} \\
M
\end{array}\right) \frac{1}{\left(\begin{array}{c}
q^{L}-M+M_{0} \\
M_{0}
\end{array}\right)} \cdot f\left(L, M_{0}, d\right) .
$$

Thus, the redundancy

$$
\begin{aligned}
r(\mathcal{C}) & =\log _{q}\left(\begin{array}{l}
q^{L} \\
M
\end{array}\right)-\log _{q}|\mathcal{C}| \\
& \geq \log _{q}\left(\begin{array}{l}
q^{L} \\
M
\end{array}\right)-\log _{q}\left(\left(\begin{array}{l}
q^{L} \\
M
\end{array}\right) \frac{1}{\left(q^{L}-M+M_{0}\right)} \cdot f\left(L, M_{0}, d\right)\right) \\
& =\log _{q} \frac{\left(q^{L}-M+M_{0}\right) !}{M_{0} !\left(q^{L}-M\right) ! f\left(L, M_{0}, d\right)},
\end{aligned}
$$

where $f\left(L, M_{0}, d\right)$ is defined as in (17).

Clearly, Theorem 3 is a special case of Theorem 6 with $M=M_{0}=\left\lceil\frac{d}{L}\right\rceil$ and $d=L M_{0}$. It is still not known whether the bound given in Theorem 6 is achievable for the case that $d<L M_{0}$ or $M>M_{0}$.

\section{Constructions of Sequence-Subset Codes}

In this section, we give some constructions of sequencesubset codes. As in Section III, we will always assume that $\mathbb{A}$ is an alphabet of size $q$.

\section{A. Construction of Optimal Codes}

The following construction gives a family of optimal sequence-subset code (with respect to code size) for the special case that $d=L M$ and $M^{\frac{1}{L}}$ is an integer.

Construction 1: Suppose $d=L M, M^{\frac{1}{L}}<q$ is an integer and $N=\left\lfloor q M^{-\frac{1}{L}}\right\rfloor$. Partition $\mathbb{A}$ into $N$ mutually disjoint subsets, say $W_{1}, W_{2}, \cdots, W_{N}$, such that $\left|W_{i}\right| \geq M^{\frac{1}{L}}$, $i=1,2, \cdots, N$. For each $i \in[N]$, pick a subset $\mathbf{X}_{i}=$ $\left\{\mathbf{x}_{i, 1}, \mathbf{x}_{i, 2}, \cdots, \mathbf{x}_{i, M}\right\} \subseteq W_{i}^{L}$, and let $\mathcal{C}=\left\{\mathbf{X}_{i} ; i \in[N]\right\}$.

Theorem 7: The code $\mathcal{C}$ obtained by Construction 1 is an $(L, M, N, d)_{q}$ sequence-subset code.

Proof: Since $N=\left\lfloor q M^{-\frac{1}{L}}\right\rfloor$, we have $N \leq q M^{-\frac{1}{L}}$, and hence $N M^{\frac{1}{L}} \leq q$. The set $\mathbb{A}$ can always be partitioned into $W_{1}, W_{2}, \cdots, W_{N}$ satisfying the expected conditions. Moreover, since $\left|W_{i}^{L}\right| \geq|M|$ for all $i \in[N]$, the subsets $\mathbf{X}_{i}=\left\{\mathbf{x}_{i, 1}, \mathbf{x}_{i, 2}, \cdots, \mathbf{x}_{i, M}\right\} \subseteq W_{i}^{L}$, and hence $\mathcal{C}$, can always be constructed as in Construction 1 .

Clearly, $\mathcal{C} \subseteq \mathcal{P}\left(\mathbb{A}^{L}\right)$ is a sequence-subset code with constant codeword size $M$ and $|\mathcal{C}|=N=\left\lfloor q M^{-\frac{1}{L}}\right\rfloor$. Moreover, for any distinct $i_{1}, i_{2} \in[N]$ and any $j_{1}, j_{2} \in[M]$, since 
$W_{1}, W_{1}, \cdots, W_{N}$ are mutually disjoint, $\mathbf{x}_{i_{1}, j_{1}} \in W_{i_{1}}^{L}$ and $\mathbf{x}_{i_{2}, j_{2}} \in W_{i_{2}}^{L}$, it is easy to see that

$$
d_{\mathrm{H}}\left(\mathbf{x}_{i_{1}, j_{1}}, \mathbf{x}_{i_{2}, j_{2}}\right)=L .
$$

By (1) and (2), for any distinct $i_{1}, i_{2} \in[N]$, we have

$$
d_{\mathrm{S}}\left(\mathbf{X}_{i_{1}}, \mathbf{X}_{i_{2}}\right)=L M=d
$$

which implies that $d_{\mathrm{S}}(\mathcal{C})=d$. Thus, $\mathcal{C}$ is an $(L, M, N, d)_{q}$ sequence-subset code.

Example 3: As an illustrative example of Construction 1, we let $\mathbb{A}=\{0,1, \cdots, 15\}, L=4$ and $M=16$. Then $q=16$, $d=64, M^{\frac{1}{L}}=2$ and $N=\left\lfloor q M^{-\frac{1}{L}}\right\rfloor=8$. We partition $\mathbb{A}$ into $W_{1}, W_{2}, \cdots, W_{8}$, where $W_{i}=\{2(i-1), 2 i-1\}$, $i=1,2, \cdots, 8$. Then we can choose $\mathbf{X}_{i}=W_{i}^{4}$ and let $\mathcal{C}=$ $\left\{\mathbf{X}_{1}, \mathbf{X}_{2}, \cdots, \mathbf{X}_{8}\right\}$. For example, we have $\mathbf{X}_{1}=\{0000,0001$, 0010, 0011, 0100, 0101, 0110, 0111, 1000, 1001, 1010, 1011, $1100,1101,1110,1111\}$ and $\mathbf{X}_{2}=\{2222,2223,2232,2233$, 2322, 2323, 2332, 2333, 3222, 3223, 3232, 3233, 3322, 3323, $3332,3333\}$. Clearly, $d_{\mathrm{S}}\left(\mathbf{X}_{1}, \mathbf{X}_{2}\right)=64=d=L M$. In fact, it is easy to verify that for all distinct $i, j \in\{1,2, \cdots, 8\}$ $d_{\mathrm{S}}\left(\mathbf{X}_{i}, \mathbf{X}_{j}\right)=64=d=L M$.

Note that by Theorem 3 , if $d=L M$, then $S_{q}(L, M, d) \leq$ $\left\lfloor q M^{-\frac{1}{L}}\right\rfloor$, and so the code $\mathcal{C}$ constructed in Theorem 7 is optimal with respect to code size, and hence we have the following corollary.

Corollary 2: Suppose $d=L M$ and $M^{\frac{1}{L}}$ is an integer. We have

$$
S_{q}(L, M, d)=\left\lfloor q M^{-\frac{1}{L}}\right\rfloor
$$

For any fixed $m \in\{2, \cdots, q\}$, we can let $d=L m^{L}$, and then we have $M^{\frac{1}{L}}=\left(\frac{d}{L}\right)^{\frac{1}{L}}=m$ is an integer. In this case, $N=\left\lfloor q M^{-\frac{1}{L}}\right\rfloor=\left\lfloor\frac{q}{m}\right\rfloor$.

Remark 4: For the case that $d=L M$ but $M^{\frac{1}{L}}$ is not an integer, let $N$ be any fixed positive integer such that $\left\lfloor\frac{q}{N}\right\rfloor \geq M^{\frac{1}{L}}$. Then we can partition $\mathbb{A}$ into $N$ mutually disjoint subsets $W_{1}, W_{1}, \cdots, W_{N}$ such that for each $i \in[N]$, $\left|W_{i}\right| \geq M^{\frac{1}{L}}$. By the same construction as in Theorem 7 we can obtain an $(L, M, N, d)_{q}$ sequence-subset code. Thus, we have $N^{*} \leq S_{q}(L, M, d) \leq\left\lfloor q M^{-\frac{1}{L}}\right\rfloor$, where $N^{*}=$ $\max \left\{N ;\left\lfloor\frac{q}{N}\right\rfloor \geq M^{\frac{1}{L}}\right\}$.

\section{B. Construction Based on Binary Codes}

In the rest of this section, to distinguish from sequencesubset code (i.e., a subset of the power set $\mathcal{P}\left(\mathbb{A}^{L}\right)$ of the set $\left.\mathbb{A}^{L}\right)$, we will call any subset of $\mathbb{A}^{L}$ a conventional code. An $(L, N, d)_{q}$ conventional code is a subset of $\mathbb{A}^{L}$ with $N$ codewords and the minimum Hamming distance $d$ (recalling that $q$ is the size of the alphabet $\mathbb{A}$ ). Our following constructions of sequence-subset codes are based on conventional codes with respect to the Hamming distance.

The following construction is a modification of Construction 2 of [14].
Construction 2: Let $\mathcal{C}_{1}=\left\{\mathbf{x}_{1}, \mathbf{x}_{2}, \cdots, \mathbf{x}_{K}\right\} \subseteq \mathbb{A}^{L}$ be a conventional code over $\mathbb{A}$ and $\mathcal{C}_{2}=\left\{\mathbf{w}_{1}, \mathbf{w}_{2}, \cdots, \mathbf{w}_{N}\right\} \subseteq \mathbb{F}_{2}^{K}$ be a conventional binary code. For each $\mathbf{w}_{i} \in \mathcal{C}_{2}$, let

$$
\mathbf{X}_{i}=\left\{\mathbf{x}_{j} ; j \in \operatorname{supp}\left(\mathbf{w}_{i}\right)\right\},
$$

where $\operatorname{supp}\left(\mathbf{w}_{i}\right)=\left\{j \in[K] ; \mathbf{w}_{i}(j) \neq 0\right\}$ is the support of $\mathbf{w}_{i}$. Finally, let

$$
\mathcal{C}=\left\{\mathbf{X}_{1}, \mathbf{X}_{2}, \cdots, \mathbf{X}_{N}\right\}
$$

Then we have the following theorem.

Theorem 8: Suppose $\mathcal{C}_{1}$ has the minimum (Hamming) distance $d_{1}$ and $\mathcal{C}_{2}$ has the minimum (Hamming) distance $d_{2}$. Then the code $\mathcal{C}$ obtained by Construction 2 has sequence length $L$, code size $|\mathcal{C}|=N$, and the minimum sequencesubset distance $d_{\mathrm{S}}(\mathcal{C})$ satisfies

$$
d_{\mathrm{S}}(\mathcal{C}) \geq d_{1} \cdot\left\lceil\frac{d_{2}}{2}\right\rceil .
$$

Proof: Clearly, $\mathcal{C}$ has sequence length $L$ and code size $|\mathcal{C}|=N$. It remains to prove that $d_{\mathrm{S}}(\mathcal{C}) \geq d_{1} \cdot\left\lceil\frac{d_{2}}{2}\right\rceil$.

Let $\mathbf{X}_{i_{1}}$ and $\mathbf{X}_{i_{2}}$ be any distinct codewords of $\mathcal{C}$. We need to prove $d_{\mathrm{S}}\left(\mathbf{X}_{i_{1}}, \mathbf{X}_{i_{2}}\right) \geq d_{1} \cdot\left\lceil\frac{d_{2}}{2}\right\rceil$.

Without loss of generality, assume that $\left|\mathbf{X}_{i_{1}}\right| \leq\left|\mathbf{X}_{i_{2}}\right|$. Then we have $\left|\mathbf{X}_{i_{1}} \backslash \mathbf{X}_{i_{2}}\right| \leq\left|\mathbf{X}_{i_{2}} \backslash \mathbf{X}_{i_{1}}\right|$. To simplify notation, denote

$$
\tilde{\mathbf{X}}_{i_{1}}=\mathbf{X}_{i_{1}} \backslash \mathbf{X}_{i_{2}} \text { and } \tilde{\mathbf{X}}_{i_{2}}=\mathbf{X}_{i_{2}} \backslash \mathbf{X}_{i_{1}} .
$$

For an arbitrary injection $\chi: \tilde{\mathbf{X}}_{i_{1}} \rightarrow \tilde{\mathbf{X}}_{i_{2}}$, by (1),

$$
d_{\chi}\left(\tilde{\mathbf{X}}_{i_{1}}, \tilde{\mathbf{X}}_{i_{2}}\right)=\sum_{\mathbf{x} \in \tilde{\mathbf{X}}_{i_{1}}} d_{\mathrm{H}}(\mathbf{x}, \chi(\mathbf{x}))+L\left(\left|\tilde{\mathbf{X}}_{i_{2}}\right|-\left|\tilde{\mathbf{X}}_{i_{1}}\right|\right) .
$$

Since $\mathcal{C}_{1}$ has the minimum (Hamming) distance $d_{1}$ and by construction of $\mathcal{C}, \mathbf{x}$ and $\chi(\mathbf{x})$ are distinct codeword in $\mathcal{C}_{1}$, so

$$
\sum_{\mathbf{x} \in \tilde{\mathbf{X}}_{i_{1}}} d_{\mathrm{H}}(\mathbf{x}, \chi(\mathbf{x})) \geq\left|\tilde{\mathbf{X}}_{i_{1}}\right| \cdot d_{1} .
$$

Moreover, since $\mathcal{C}_{1} \subseteq \mathbb{A}^{L}$, then $L \geq d_{1}$. Hence, (19) implies that

$$
\begin{aligned}
d_{\chi}\left(\tilde{\mathbf{X}}_{i_{1}}, \tilde{\mathbf{X}}_{i_{2}}\right) & \geq\left|\tilde{\mathbf{X}}_{i_{1}}\right| \cdot d_{1}+d_{1}\left(\left|\tilde{\mathbf{X}}_{i_{2}}\right|-\left|\tilde{\mathbf{X}}_{i_{1}}\right|\right) \\
& =d_{1} \cdot\left|\tilde{\mathbf{X}}_{i_{2}}\right| \\
& =d_{1} \cdot\left|\mathbf{X}_{i_{2}} \backslash \mathbf{X}_{i_{1}}\right| .
\end{aligned}
$$

By the construction of $\mathcal{C}, \mathbf{X}_{i_{1}}=\left\{\mathbf{x}_{j} ; j \in \operatorname{supp}\left(\mathbf{w}_{i_{1}}\right)\right\}$ and $\mathbf{X}_{i_{2}}=\left\{\mathbf{x}_{j} ; j \in \operatorname{supp}\left(\mathbf{w}_{i_{2}}\right)\right\}$ for some distinct $\mathbf{w}_{i_{1}}, \mathbf{w}_{i_{2}} \in \mathcal{C}_{2}$. Then we have

$$
\left|\mathbf{X}_{i_{1}} \backslash \mathbf{X}_{i_{2}}\right|+\left|\mathbf{X}_{i_{2}} \backslash \mathbf{X}_{i_{1}}\right|=d_{\mathrm{H}}\left(\mathbf{w}_{i_{1}}, \mathbf{w}_{i_{2}}\right) \geq d_{2}
$$

where $d_{2}$ is the minimum (Hamming) distance of $\mathcal{C}_{2}$. Note that $\left|\mathbf{X}_{i_{1}} \backslash \mathbf{X}_{i_{2}}\right| \leq\left|\mathbf{X}_{i_{2}} \backslash \mathbf{X}_{i_{1}}\right|$. Then by the above equation, we have $\left|\mathbf{X}_{i_{2}} \backslash \mathbf{X}_{i_{1}}\right| \geq \frac{d_{2}}{2}$. Moreover, since $\left|\mathbf{X}_{i_{2}} \backslash \mathbf{X}_{i_{1}}\right|$ is an integer, so

$$
\left|\mathbf{X}_{i_{2}} \backslash \mathbf{X}_{i_{1}}\right| \geq\left\lceil\frac{d_{2}}{2}\right\rceil
$$

Combining this with 20, we have

$$
d_{\chi}\left(\tilde{\mathbf{X}}_{i_{1}}, \tilde{\mathbf{X}}_{i_{2}}\right) \geq d_{1} \cdot\left\lceil\frac{d_{2}}{2}\right\rceil \text {. }
$$


Note that $\chi: \mathbf{X}_{i_{1}} \backslash \mathbf{X}_{i_{2}} \rightarrow \mathbf{X}_{i_{2}} \backslash \mathbf{X}_{i_{1}}$ is an arbitrary injection. By Definition 1 and Corollary 1 we have

$$
d_{\mathrm{S}}\left(\mathbf{X}_{i_{1}}, \mathbf{X}_{i_{2}}\right)=d_{\mathrm{S}}\left(\mathbf{X}_{i_{1}} \backslash \mathbf{X}_{i_{2}}, \mathbf{X}_{i_{2}} \backslash \mathbf{X}_{i_{1}}\right) \geq d_{1} \cdot\left\lceil\frac{d_{2}}{2}\right\rceil,
$$

which completes the proof.

Example 4: Let $\mathcal{C}_{1}$ be a binary $[5,3]$ linear code with generator matrix $G_{1}$ and $\mathcal{C}_{2}$ be a binary linear $[8,3]$ code with generator matrix $G_{2}$, where

$$
G_{1}=\left(\begin{array}{ccccc}
1 & 0 & 0 & 1 & 0 \\
0 & 1 & 0 & 0 & 1 \\
0 & 0 & 1 & 1 & 1
\end{array}\right)
$$

and

$$
G_{2}=\left(\begin{array}{cccccccc}
1 & 0 & 0 & 1 & 1 & 1 & 0 & 0 \\
0 & 1 & 0 & 0 & 0 & 1 & 1 & 1 \\
0 & 0 & 1 & 1 & 0 & 1 & 0 & 1
\end{array}\right) .
$$

We have $L=5, K=N=8$, the minimum distance of $\mathcal{C}_{1}$ is $d_{1}=2$ and the minimum distance of $\mathcal{C}_{2}$ is $d_{2}=4$. Denote $\mathcal{C}_{1}=\left\{\mathbf{x}_{1}, \mathbf{x}_{2}, \cdots, \mathbf{x}_{8}\right\}$, where $\mathbf{x}_{1}=00000$, $\mathbf{x}_{2}=10010, \mathbf{x}_{3}=01001, \mathbf{x}_{4}=00111, \mathbf{x}_{5}=11011$, $\mathbf{x}_{6}=10101, \mathbf{x}_{7}=01110, \mathbf{x}_{8}=11100$. Then by Construction 2 , for each codeword $\mathbf{w} \in \mathcal{C}_{2}$, we can obtain a subset $\mathbf{X}_{\mathbf{w}}=\left\{\mathbf{x}_{j} ; j \in \operatorname{supp}(\mathbf{w})\right\} \subseteq \mathbb{F}_{2}^{5}$, and this gives a code $\mathcal{C}=\left\{\mathbf{X}_{\mathbf{w}} ; \mathbf{w} \in \mathcal{C}_{2}\right\}$. For example, for $\mathbf{w}=10011100$, we have $\mathbf{X}_{\mathbf{w}}=\left\{\mathbf{x}_{1}, \mathbf{x}_{4}, \mathbf{x}_{5}, \mathbf{x}_{6}\right\}=\{00000,00111,11011,10101\}$; for $\mathbf{w}^{\prime}=11101110$, we have $\mathbf{X}_{\mathbf{w}^{\prime}}=\left\{\mathbf{x}_{1}, \mathbf{x}_{2}, \mathbf{x}_{3}, \mathbf{x}_{5}, \mathbf{x}_{6}, \mathbf{x}_{7}\right\}=$ $\{00000,10010,01001,11011,10101,01110\}$. By Corollary 1 we have $d_{\mathbf{S}}\left(\mathbf{X}_{\mathbf{w}}, \mathbf{X}_{\mathbf{w}^{\prime}}\right)=d_{\mathbf{S}}\left(\mathbf{X}_{\mathbf{w}}^{\prime}, \mathbf{X}_{\mathbf{w}^{\prime}}^{\prime}\right)$, where $\mathbf{X}_{\mathbf{w}}^{\prime}=\mathbf{X}_{\mathbf{w}} \backslash \mathbf{X}_{\mathbf{w}^{\prime}}=$ $\left\{\mathbf{x}_{4}\right\}=\{00111\}$ and $\mathbf{X}_{\mathbf{w}^{\prime}}^{\prime}=\mathbf{X}_{\mathbf{w}^{\prime}} \backslash \mathbf{X}_{\mathbf{w}^{\prime}}=\left\{\mathbf{x}_{2}, \mathbf{x}_{3}, \mathbf{x}_{7}\right\}=$ $\{10010,01001,01110\}$. Further, by (1) and (2), we can obtain $d_{\mathrm{S}}\left(\mathbf{X}_{\mathbf{w}}^{\prime}, \mathbf{X}_{\mathbf{w}^{\prime}}^{\prime}\right)=2+2 L=12$. Note that $\left|\mathbf{X}_{\mathbf{w}}\right| \neq\left|\mathbf{X}_{\mathbf{w}^{\prime}}\right|$, so the code $\mathcal{C}$ is not a constant-codeword-size code.

Remark 5: The code $\mathcal{C}$ obtained by Construction 2 may or may not have constant codeword size, depending on whether $\mathcal{C}_{2}$ is a constant weight binary code. In fact, $\mathcal{C}$ is a constantcodeword-size code if and only if $\mathcal{C}_{2}$ is a constant-weight binary code.

To compare $|\mathcal{C}|$ with the bound in Theorem 6, we let $\mathcal{C}_{1}$ be an $\left(L, K, d_{1}\right)$ code over $\mathbb{A}$ and $\mathcal{C}_{2}$ be a $(K, 2 \delta, M)$ binary constant-weight code such that $L>d_{1}>\left(1-\frac{1}{q}\right) L$ and $\delta<q$. Then by construction 2 and Theorem 8 , we can obtain a sequence-subset code $\mathcal{C}$ with sequence length $L$, code size $|\mathcal{C}|=\left|\mathcal{C}_{2}\right|$, constant codeword size $M$, and minimum distance $d_{\mathrm{S}}(\mathcal{C}) \geq d=d_{1} \delta$. For $\mathcal{C}_{1}$, by the Plotking bound [20],

$$
K \leq K_{0} \triangleq\left\lfloor\frac{d_{1}}{d_{1}-r L}\right\rfloor,
$$

where $r=1-\frac{1}{q}$. Then we have

$$
\left|\mathcal{C}_{2}\right| \leq A(K, 2 \delta, M) \leq A\left(K_{0}, 2 \delta, M\right),
$$

where for any $n, d$ and $w, A(n, d, w)$ denotes the maximum number of codewords of a binary constant weight code of length $n$, minimum Hamming distance $d$ and constant weight $w$. By the Johnson bound [21],

$$
A\left(K_{0}, 2 \delta, M\right) \leq\left\lfloor\frac{K_{0}}{M}\left\lfloor\frac{K_{0}-1}{M-1} \cdots\left\lfloor\frac{K_{0}-M+\delta}{\delta}\right\rfloor \ldots\right\rfloor\right\rfloor .
$$

Then we have

$$
|\mathcal{C}|=\left|\mathcal{C}_{2}\right| \leq\left\lfloor\frac{K_{0}}{M}\left\lfloor\frac{K_{0}-1}{M-1} \cdots\left\lfloor\frac{K_{0}-M+\delta}{\delta}\right\rfloor \ldots\right\rfloor\right\rfloor .
$$

On the other hand, since $L>d_{1}>\left(1-\frac{1}{q}\right) L$ and $\delta<q$, we have $M_{0}=\left\lceil\frac{d_{1} \delta}{L}\right\rceil=\delta$ and $\left(1-\frac{1}{q}\right) L M_{0}<d_{1} \delta<L M_{0}$. According to Theorem 6 we have

$$
\begin{aligned}
|\mathcal{C}| & \leq\left\lfloor\frac{q^{L}}{M}\left\lfloor\frac{q^{L}-1}{M-1} \cdots\left\lfloor\frac{q^{L}-M+M_{0}+1}{M_{0}+1} \frac{d_{1} \delta}{d_{1} \delta-r L \delta}\right\rfloor \cdots\right\rfloor\right\rfloor \\
& =\left\lfloor\frac{q^{L}}{M}\left\lfloor\frac{q^{L}-1}{M-1} \cdots\left\lfloor\frac{q^{L}-M+\delta+1}{\delta+1} K_{0}\right\rfloor \ldots\right\rfloor\right\rfloor .
\end{aligned}
$$

Note that

$$
\begin{aligned}
& \left.\left\lfloor\frac{q^{L}}{M}\left\lfloor\frac{q^{L}-1}{M-1} \ldots\left\lfloor\frac{q^{L}-M+\delta+1}{\delta+1} K_{0}\right\rfloor \ldots\right\rfloor\right\rfloor\right\rfloor \\
& >\left\lfloor\frac{K_{0}}{M}\left\lfloor\frac{K_{0}-1}{M-1} \ldots\left\lfloor\frac{K_{0}-M+\delta}{\delta}\right\rfloor \ldots\right\rfloor\right\rfloor,
\end{aligned}
$$

which can be obtained from the simple facts that $K_{0}=$ $\left\lfloor\frac{d_{1}}{d_{1}-r L}\right\rfloor<q^{L}$ and $K_{0} \geq \frac{K_{0}-M+\delta}{\delta}$ (noticing that $\delta=M_{0} \leq$ $M)$. Hence, the size of codes obtained from Construction 2 does not achieve the bound in Theorem 6

\section{Construction Based on q-ary Codes $(q \geq 2)$}

In this subsection, we present a construction based on $q$-ary codes, where $q \geq 2$.

Construction 3: Let $\mathbb{A}$ and $\mathbb{B}$ be two alphabets of size $q$ and $\tilde{q}$, respectively. Let $\mathcal{C}_{1}$ be an $\left(L, M \tilde{q}, d_{1}\right)_{q}$ conventional code over $\mathbb{A}$ and $\mathcal{C}_{2}$ be an $\left(M, N, d_{2}\right)_{\tilde{q}}$ conventional code over $\mathbb{B}$. The $M \tilde{q}$ codewords of $\mathcal{C}_{1}$ can be indexed as

$$
\mathcal{C}_{1}=\left\{\mathbf{x}_{i, j}: i \in[M], j \in \mathbb{B}\right\} .
$$

From each $\mathbf{c}=\left(c_{1}, c_{2}, \cdots, c_{M}\right) \in \mathcal{C}_{2}$, we can obtain a subset

$$
\mathbf{X}_{\mathbf{c}}=\left\{\mathbf{x}_{1, c_{1}}, \mathbf{x}_{2, c_{2}}, \cdots, \mathbf{x}_{M, c_{M}}\right\} \subseteq \mathcal{C}_{1},
$$

and finally, let

$$
\mathcal{C}=\left\{\mathbf{X}_{\mathbf{c}} ; \mathbf{c} \in \mathcal{C}_{2}\right\} .
$$

Then $\mathcal{C}$ is a sequence-subset code over $\mathbb{A}$ and we have the following theorem.

Theorem 9: The code $\mathcal{C}$ obtained by Construction 3 has sequence length $L$, constant codeword size $M$, code size $|\mathcal{C}|=N$, and minimum sequence-subset distance

$$
d_{\mathrm{S}}(\mathcal{C}) \geq d_{1} d_{2} .
$$

Proof: From the construction it is easy to see that $\mathcal{C}$ has sequence length $L$, constant codeword size $M$ and code size $|\mathcal{C}|=N$. It remains to prove that $d_{\mathbf{S}}(\mathcal{C}) \geq d_{1} d_{2}$, that is, $d_{\mathrm{S}}\left(\mathbf{X}_{\mathbf{c}}, \mathbf{X}_{\mathbf{c}^{\prime}}\right) \geq d_{1} d_{2}$ for any distinct $\mathbf{X}_{\mathbf{c}}$ and $\mathbf{X}_{\mathbf{c}^{\prime}}$ in $\mathcal{C}$, where $\mathbf{c}=\left(c_{1}, c_{2}, \cdots, c_{M}\right)$ and $\mathbf{c}^{\prime}=\left(c_{1}^{\prime}, c_{2}^{\prime}, \cdots, c_{M}^{\prime}\right)$ are any pair of distinct codewords in $\mathcal{C}_{2}$.

Let $A$ be the set of all $i \in[M]$ such that $c_{i} \neq c_{i}^{\prime}$. Since $\mathcal{C}_{2}$ has the minimum (Hamming) distance $d_{2}$, then

$$
|A|=d_{\mathrm{H}}\left(\mathbf{c}, \mathbf{c}^{\prime}\right) \geq d_{2} .
$$


Denote

$$
\tilde{\mathbf{X}}_{\mathbf{c}}=\left\{\mathbf{x}_{i, c_{i}} ; i \in A\right\} \text { and } \tilde{\mathbf{X}}_{\mathbf{c}^{\prime}}=\left\{\mathbf{x}_{i, c_{i}^{\prime}} ; i \in A\right\} .
$$

Then by the construction, we have

$$
\tilde{\mathbf{X}}_{\mathbf{c}}=\mathbf{X}_{\mathbf{c}} \backslash \mathbf{X}_{\mathbf{c}^{\prime}} \text { and } \tilde{\mathbf{X}}_{\mathbf{c}^{\prime}}=\mathbf{X}_{\mathbf{c}^{\prime}} \backslash \mathbf{X}_{\mathbf{c}}
$$

By Corollary 1] it suffices to prove that $d_{\mathrm{S}}\left(\tilde{\mathbf{X}}_{\mathbf{c}}, \tilde{\mathbf{X}}_{\mathbf{c}^{\prime}}\right) \geq d_{1} d_{2}$.

Note that $\left|\tilde{\mathbf{X}}_{\mathbf{c}}\right|=\left|\tilde{\mathbf{X}}_{\mathbf{c}^{\prime}}\right|=|A|$ and $\tilde{\mathbf{X}}_{\mathbf{c}} \cap \tilde{\mathbf{X}}_{\mathbf{c}^{\prime}}=\emptyset$. Then for any injection $\chi: \tilde{\mathbf{X}}_{\mathbf{c}} \rightarrow \tilde{\mathbf{X}}_{\mathbf{c}^{\prime}}$, we have

$$
\begin{aligned}
d_{\chi}\left(\tilde{\mathbf{X}}_{\mathbf{c}}, \tilde{\mathbf{X}}_{\mathbf{c}^{\prime}}\right) & =\sum_{\mathbf{x} \in \tilde{\mathbf{X}}_{\mathbf{c}}} d_{\mathrm{H}}(\mathbf{x}, \chi(\mathbf{x})) \\
& \geq|A| \cdot d_{1} \\
& \geq d_{1} d_{2},
\end{aligned}
$$

where the equality comes from (1), the first inequality comes from the assumption that $\mathcal{C}_{1}$ has the minimum (Hamming) distance $d_{1}$, and the second inequality comes from the fact that $|A| \geq d_{2}$. By Definition 1 $d_{\mathrm{S}}\left(\tilde{\mathbf{X}}_{\mathbf{c}}, \tilde{\mathbf{X}}_{\mathbf{c}^{\prime}}\right) \geq d_{1} d_{2}$, and hence by Corollary $1 d_{\mathbf{S}}\left(\mathbf{X}_{\mathbf{c}}, \mathbf{X}_{\mathbf{c}^{\prime}}\right) \geq d_{1} d_{2}$. Since $\mathbf{X}_{\mathbf{c}}$ and $\mathbf{X}_{\mathbf{c}^{\prime}}$ are any pair of distinct codewords in $\mathcal{C}$, we have $d_{\mathrm{S}}(\mathcal{C}) \geq d_{1} d_{2}$, which completes the proof.

Example 5: For illustration of Construction 3, we give a simple example with $M=4$ and $q=\tilde{q}=2$. Let $\mathcal{C}_{1}$ be the $[5,3]$ binary linear code given in Example 4 and $\mathcal{C}_{2}=$ $\left\{\mathbf{c}_{1}, \mathbf{c}_{2}, \mathbf{c}_{3}, \mathbf{c}_{4}\right\}$ be a binary code, where $\mathbf{c}_{1}=0000, \mathbf{c}_{2}=0101$, $\mathbf{c}_{3}=1010$ and $\mathbf{c}_{4}=1111$. The codewords of $\mathcal{C}_{1}$ can be denoted as $\mathbf{x}_{i, j}, i=1,2,3,4, j=0,1$ such that $\mathbf{x}_{1,0}=00000$, $\mathbf{x}_{2,0}=01001, \mathbf{x}_{3,0}=10010, \mathbf{x}_{4,0}=11011, \mathbf{x}_{1,1}=00111$, $\mathbf{x}_{2,1}=01110, \mathbf{x}_{3,1}=10101$ and $\mathbf{x}_{4,1}=11100$. Then by Construction 3 , we have $\mathcal{C}=\left\{\mathbf{X}_{\mathbf{c}_{1}}, \mathbf{X}_{\mathbf{c}_{2}}, \mathbf{X}_{\mathbf{c}_{3}}, \mathbf{X}_{\mathbf{c}_{4}}\right\}$, where $\mathbf{X}_{\mathbf{c}_{1}}=\left\{\mathbf{x}_{1,0}, \mathbf{x}_{2,0}, \mathbf{x}_{3,0}, \mathbf{x}_{4,0}\right\}, \mathbf{X}_{\mathbf{c}_{2}}=\left\{\mathbf{x}_{1,0}, \mathbf{x}_{2,1}, \mathbf{x}_{3,0}, \mathbf{x}_{4,1}\right\}$, $\mathbf{X}_{\mathbf{c}_{3}}=\left\{\mathbf{x}_{1,1}, \mathbf{x}_{2,0}, \mathbf{x}_{3,1}, \mathbf{x}_{4,0}\right\}$ and $\mathbf{X}_{\mathbf{c}_{4}}=\left\{\mathbf{x}_{1,1}, \mathbf{x}_{2,1}, \mathbf{x}_{3,1}, \mathbf{x}_{4,1}\right\}$. It is easy to see that $d_{2}=2$ and $d_{\mathrm{S}}\left(\mathbf{X}_{\mathbf{c}}, \mathbf{X}_{\mathbf{c}^{\prime}}\right) \geq d_{1} d_{2}=4$ for any distinct $c, c^{\prime} \in \mathcal{C}_{2}$. For example, by Corollary 1 $d_{\mathrm{S}}\left(\mathbf{X}_{\mathbf{c}_{1}}, \mathbf{X}_{\mathbf{c}_{2}}\right)=d_{\mathrm{S}}\left(\left\{\mathbf{x}_{2,0}, \mathbf{x}_{4,0}\right\},\left\{\mathbf{x}_{2,1}, \mathbf{x}_{4,1}\right\}\right)=6$.

The following is a more general example of Construction 3.

Example 6: Let $\mathcal{C}_{1}$ be an $\left[L, k, d_{1}\right]_{q}$ linear code such that the first $k$ symbols of the codewords of $\mathcal{C}_{1}$ are the information symbols. For any given integer $r$ such that $1 \leq r<k$, let $\tilde{q}=q^{r}$ and $M=q^{s}$, where $s=k-r$. Note that there exists a bijection $\pi:[M] \rightarrow \mathbb{F}_{q}^{s}$. Moreover, fixing a basis, each element of $\mathbb{F}_{q^{r}}$ can be uniquely represented as a vector in $\mathbb{F}_{q}^{r}$, so we can identify each element of $\mathbb{F}_{q^{r}}$ as a vector in $\mathbb{F}_{q}^{r}$. Then for each $i \in[M]$ and each $j \in \mathbb{F}_{q^{r}}$, we can let

$$
\begin{aligned}
& \mathbf{x}_{i, j}=\left(x_{1}, x_{2}, \cdots, x_{L}\right): \\
& \left(x_{1}, x_{2}, \cdots, x_{s}\right)=\pi(i) \text { and }\left(x_{s+1}, \cdots, x_{k}\right)=j .
\end{aligned}
$$

Now, let $\mathcal{C}_{2}$ be an $\left[M, K, d_{2}\right]_{q^{r}}$ linear code, where $K \in$ $[M]$ is another design parameter. Then for each $\mathbf{c}=$ $\left(c_{1}, c_{2}, \cdots, c_{M}\right) \in \mathcal{C}_{2}$, we can obtain

$$
\mathbf{X}_{\mathbf{c}}=\left\{\mathbf{x}_{1, c_{1}}, \mathbf{x}_{2, c_{2}}, \cdots, \mathbf{x}_{M, c_{M}}\right\} \subseteq \mathcal{C}_{1},
$$

that is, for each $i \in[M], \mathbf{x}_{i, c_{i}}=\left(x_{1}, x_{2}, \cdots, x_{L}\right)$ such that

$$
\left(x_{1}, x_{2}, \cdots, x_{s}\right)=\pi(i) \text { and }\left(x_{s+1}, \cdots, x_{k}\right)=c_{i} .
$$

Finally, we have

$$
\mathcal{C}=\left\{\mathbf{X}_{\mathbf{c}} ; \mathbf{c} \in \mathcal{C}_{2}\right\} .
$$

By Theorem 10, $\mathcal{C}$ is a sequence-subset code of sequence length $L$, constant codeword size $M=q^{s}$, code size $|\mathcal{C}|=\left|\mathcal{C}_{2}\right|=q^{r K}$, and minimum sequence-subset distance $d_{\mathrm{S}}(\mathcal{C}) \geq d_{1} d_{2}$.

As a special case of Example 6 suppose $q \geq L-1$ and $s \leq \frac{k}{2}$. We can let $\mathcal{C}_{1}$ be an $[L, k, L-k+1]_{q}$ MDS code and $\mathcal{C}_{2}$ be a $\left[q^{s}, K, q^{s}-K+1\right]_{q^{r}}$ MDS code. Then the minimum distance of $\mathcal{C}$ satisfies $d_{\mathrm{S}}(\mathcal{C}) \geq(L-k+1)\left(q^{s}-K+1\right)$. This special case is essentially similar to the method used in [7].

Let $\mathcal{C}$ be a sequence-subset code obtained by Construction 3 . To compare $|\mathcal{C}|$ with the bound in Theorem 4 , we consider $\tilde{q}>$ $q, L>d_{1}>\left(1-\frac{1}{q}\right)\left(1-\frac{1}{\tilde{q}}\right)^{-1} L$ and $M>d_{2}>\left(1-\frac{1}{\tilde{q}}\right) M$. Then $L>d_{1}>\left(1-\frac{1}{q}\right) L$ and $L M>d_{1} d_{2}>\left(1-\frac{1}{q}\right) L M$. By the Plotking bound [20], we have

$$
M \tilde{q}=\left|\mathcal{C}_{1}\right| \leq \frac{d_{1}}{d_{1}-r L},
$$

where $r=1-\frac{1}{q}$, and

$$
N=\left|\mathcal{C}_{2}\right| \leq \frac{d_{2}}{d_{2}-\tilde{r} M},
$$

where $\tilde{r}=1-\frac{1}{\tilde{q}}$, On the other hand, by Theorem 4

$$
|\mathcal{C}|=N \leq \frac{d_{1} d_{2}}{d_{1} d_{2}-r L M} .
$$

By (22), we have

$$
\frac{r L}{d_{1}}>1-\frac{1}{M \tilde{q}} \geq 1-\frac{1}{\tilde{q}}=\tilde{r}
$$

which implies that

$$
1-\frac{\tilde{r} M}{d_{2}}>1-\frac{r L M}{d_{1} d_{2}},
$$

and so

$$
\frac{d_{2}}{d_{2}-\tilde{r} M}<\frac{d_{1} d_{2}}{d_{1} d_{2}-r L M} .
$$

By (23) and (24), $|\mathcal{C}|$ does not achieve the bound in Theorem 4

\section{Construction Based on Sequence Index}

In this subsection, if $\mathbf{x}=(\mathbf{x}(1), \mathbf{x}(2), \cdots, \mathbf{x}(L)) \in \mathbb{A}^{L}$ and $I=\left\{i_{1}, i_{2}, \cdots, i_{m}\right\} \subseteq[L]$ such that $i_{1}<i_{2}<\cdots<i_{m}$, then we denote $\mathbf{x}(I)=\left(\mathbf{x}\left(i_{1}\right), \mathbf{x}\left(i_{2}\right), \cdots, \mathbf{x}\left(i_{m}\right)\right)$.

The construction given in this subsection is a slight improvement of the Construction 1 of [14].

Construction 4: Let $\mathcal{C}_{1}=\left\{\mathbf{s}_{1}, \mathbf{s}_{2}, \cdots, \mathbf{s}_{M}\right\} \subseteq \mathbb{A}^{L_{1}}$ be a conventional code over $\mathbb{A}$ with block length $L_{1}$ and the minimum (Hamming) distance $d_{1}$, and $\mathcal{C}_{2}=\left\{\mathbf{u}_{1}, \mathbf{u}_{2}, \cdots, \mathbf{u}_{N}\right\} \subseteq$ $\mathbb{A}^{d_{1} M}$ be a conventional code over $\mathbb{A}$ with block length $d_{1} M$ and the minimum (Hamming) distance $d_{2}$. For each $j \in[M]$, let

$$
I_{j}=\left\{\ell \in \mathbb{Z} ;(j-1) d_{1}<\ell \leq j d_{1}\right\}
$$


and for each $i \in[N]$, let

$$
\mathbf{X}_{i}=\left\{\mathbf{x}_{i, 1}, \mathbf{x}_{i, 2}, \cdots, \mathbf{x}_{i, M}\right\}
$$

such that for each $j \in[M]$,

$$
\mathbf{x}_{i, j}=\left(\mathbf{s}_{j}, \mathbf{u}_{i}\left(I_{j}\right)\right) .
$$

Finally, let

$$
\mathcal{C}=\left\{\mathbf{X}_{i} ; i \in[N]\right\} .
$$

Then $\mathcal{C}$ is a sequence-subset code over $\mathbb{A}$. In this construction, each codeword $\mathbf{s}_{j}$ of $\mathcal{C}_{1}$ serves as an index of the sequence $\mathbf{x}_{i, j}$ of the codeword $\mathbf{X}_{i}$, and $\mathbf{u}_{i}\left(I_{j}\right)$ is the information part of $\mathbf{x}_{i, j}$. For this reason, this construction is called a construction based on sequence index. Moreover, we have the following theorem.

Theorem 10: The code $\mathcal{C}$ obtained by Construction 4 has sequence length $L=L_{1}+d_{1}$, constant codeword size $M$, code size $|\mathcal{C}|=N$, and minimum sequence-subset distance

$$
d_{\mathrm{S}}(\mathcal{C}) \geq d_{2} .
$$

Proof: Clearly, $\mathcal{C}$ has sequence length $L=L_{1}+d_{1}$, constant codeword size $M$ and code size $|\mathcal{C}|=N$. It remains to prove that $d_{\mathrm{S}}(\mathcal{C}) \geq d_{2}$.

Let $i_{1}, i_{2} \in[N]$ be any two distinct elements of $[N]$, we need to prove that $d_{\mathrm{S}}\left(\mathbf{X}_{i_{1}}, \mathbf{X}_{i_{2}}\right) \geq d_{2}$, where $\mathbf{X}_{i_{1}}=$ $\left\{\mathbf{x}_{i_{1}, 1}, \mathbf{x}_{i_{1}, 2}, \cdots, \mathbf{x}_{i_{1}, M}\right\}$ and $\mathbf{X}_{i_{2}}=\left\{\mathbf{x}_{i_{2}, 1}, \mathbf{x}_{i_{2}, 2}, \cdots, \mathbf{x}_{i_{2}, M}\right\}$. For any permutation ${ }^{3} \chi:[M] \rightarrow[M]$, let

$$
\mathcal{N}=\{j \in[M] ; \chi(j)=j\}
$$

and

$$
\tilde{\mathcal{N}}=\{j \in[M] ; \chi(j) \neq j\} .
$$

Then $\mathcal{N} \cap \tilde{\mathcal{N}}=\emptyset$ and $\mathcal{N} \cup \tilde{\mathcal{N}}=[M]$. Moreover, by (1), we have

$$
\begin{aligned}
d_{\chi}\left(\mathbf{X}_{i_{1}}, \mathbf{X}_{i_{2}}\right) & =\sum_{j=1}^{M} d_{\mathrm{H}}\left(\mathbf{x}_{i_{1}, j}, \mathbf{x}_{i_{2}, \chi(j)}\right) \\
& =\sum_{j \in \mathcal{N}} d_{\mathrm{H}}\left(\mathbf{x}_{i_{1}, j}, \mathbf{x}_{i_{2}, \chi(j)}\right)+\sum_{j \in \tilde{\mathcal{N}}} d_{\mathrm{H}}\left(\mathbf{x}_{i_{1}, j}, \mathbf{x}_{i_{2}, \chi(j)}\right. \\
& =\sum_{j \in \mathcal{N}} d_{\mathrm{H}}\left(\mathbf{x}_{i_{1}, j}, \mathbf{x}_{i_{2}, j}\right)+\sum_{j \in \tilde{\mathcal{N}}} d_{\mathrm{H}}\left(\mathbf{x}_{i_{1}, j}, \mathbf{x}_{i_{2}, \chi(j)} .\right.
\end{aligned}
$$

We will estimate the two terms of the right side of Equation (26) separately.

First, by the construction, we have

$$
\begin{aligned}
\sum_{j=1}^{M} d_{\mathrm{H}}\left(\mathbf{x}_{i_{1}, j}, \mathbf{x}_{i_{2}, j}\right) & =\sum_{j=1}^{M} d_{\mathrm{H}}\left(\mathbf{u}_{i_{1}}\left(I_{j}\right), \mathbf{u}_{i_{2}}\left(I_{j}\right)\right) \\
& =d_{\mathrm{H}}\left(\mathbf{u}_{i_{1}}, \mathbf{u}_{i_{2}}\right)=d_{2} .
\end{aligned}
$$

Moreover, since for each $i \in[N]$ and $j \in[M], \mathbf{u}_{i}\left(I_{j}\right)$ has length $d_{1}$, then again by construction of $\mathcal{C}$, we have

$$
d_{\mathrm{H}}\left(\mathbf{x}_{i_{1}, j}, \mathbf{x}_{i_{2}, j}\right)=d_{\mathrm{H}}\left(\mathbf{u}_{i_{1}}\left(I_{j}\right), \mathbf{u}_{i_{2}}\left(I_{j}\right)\right) \leq d_{1} .
$$

${ }^{3}$ Note that any bijection between $\mathbf{X}_{i_{1}}$ and $\mathbf{X}_{i_{2}}$ can be uniquely represented by a permutation on the index set $[M]$, so when applying 11 to the pair $\left\{\mathbf{X}_{i_{1}}, \mathbf{X}_{i_{2}}\right\}$, we can use permutations on $[M]$ to replace bijections between $\mathbf{X}_{i_{1}}$ and $\mathbf{X}_{i_{2}}$.
Hence, we obtain

$$
\begin{aligned}
\sum_{j \in \mathcal{N}} d_{\mathrm{H}}\left(\mathbf{x}_{i_{1}, j}, \mathbf{x}_{i_{2}, j}\right)= & \sum_{j=1}^{M} d_{\mathrm{H}}\left(\mathbf{x}_{i_{1}, j}, \mathbf{x}_{i_{2}, j}\right)-\sum_{j \in \tilde{\mathcal{N}}} d_{\mathrm{H}}\left(\mathbf{x}_{i_{1}, j}, \mathbf{x}_{i_{2}, j}\right) \\
= & \sum_{j=1}^{M} d_{\mathrm{H}}\left(\mathbf{u}_{i_{1}}\left(I_{j}\right), \mathbf{u}_{i_{2}}\left(I_{j}\right)\right) \\
& -\sum_{j \in \tilde{\mathcal{N}}} d_{\mathrm{H}}\left(\mathbf{u}_{i_{1}}\left(I_{j}\right), \mathbf{u}_{i_{2}}\left(I_{j}\right)\right) \\
\geq & d_{2}-|\tilde{\mathcal{N}}| \cdot d_{1} .
\end{aligned}
$$

Second, since $\mathcal{C}_{1}$ has the minimum (Hamming) distance $d_{1}$, then by construction of $\mathcal{C}$, we have

$$
\sum_{j \in \tilde{\mathcal{N}}} d_{\mathrm{H}}\left(\mathbf{x}_{i_{1}, j}, \mathbf{x}_{i_{2}, \chi(j)} \geq \sum_{j \in \tilde{\mathcal{N}}} d_{\mathrm{H}}\left(\mathbf{s}_{j}, \mathbf{s}_{\chi(j)}\right) \geq|\tilde{\mathcal{N}}| \cdot d_{1} .\right.
$$

Combining the above two inequalities with 26, we obtain

$$
\begin{aligned}
d_{\chi}\left(\mathbf{X}_{i_{1}}, \mathbf{X}_{i_{2}}\right) & =\sum_{j \in \mathcal{N}} d_{\mathrm{H}}\left(\mathbf{x}_{i_{1}, j}, \mathbf{x}_{i_{2}, j}\right)+\sum_{j \in \tilde{\mathcal{N}}} d_{\mathrm{H}}\left(\mathbf{x}_{i_{1}, j}, \mathbf{x}_{i_{2}, \chi(j)}\right. \\
& \geq\left(d_{2}-|\tilde{\mathcal{N}}| \cdot d_{1}\right)+|\tilde{\mathcal{N}}| \cdot d_{1} \\
& =d_{2} .
\end{aligned}
$$

Since $\chi:[M] \rightarrow[M]$ is an arbitrary bijection, then by Definition 1, we have

$$
d_{\mathrm{S}}\left(\mathbf{X}_{i_{1}}, \mathbf{X}_{i_{2}}\right) \geq d_{2}
$$

Moreover, since $i_{1}$ and $i_{2}$ are any two distinct elements of $[N]$, so we have

$$
d_{\mathrm{S}}(\mathcal{C}) \geq d_{2}
$$

which completes the proof.

Example 7: Let $\mathbb{A}=\mathbb{F}_{2}, \mathcal{C}_{1}=\left\{\mathbf{s}_{1}, \mathbf{s}_{2}\right\}$ and $\mathcal{C}_{2}=$ $\left\{\mathbf{u}_{1}, \mathbf{u}_{2}, \mathbf{u}_{3}\right\}$, where $\mathbf{s}_{1}=0000, \mathbf{s}_{2}=1111, \mathbf{u}_{1}=00000000$, $\mathbf{u}_{2}=11111000$ and $\mathbf{u}_{3}=01010111$. We can check that $L_{1}=d_{1}=4, M=2, N=3$ and $d_{2}=5$. We can divide each $\mathbf{u}_{i}$ into $M=2$ segments, each of length $L_{1}=4$, and denote each $\mathbf{u}_{i}=\mathbf{u}_{i, 1} \mathbf{u}_{i, 2}$. For example, $\mathbf{u}_{3,1}=0101$ and $\mathbf{u}_{3,2}=$ 0111. By Construction 4, we can obtain $\mathcal{C}=\left\{\mathbf{X}_{1}, \mathbf{X}_{2}, \mathbf{X}_{3}\right\}$, where $\mathbf{X}_{1}=\left\{\mathbf{s}_{1} \mathbf{u}_{1,1}, \mathbf{s}_{2} \mathbf{u}_{1,2}\right\}=\{00000000,11110000\}$, $\mathbf{X}_{2}=\left\{\mathbf{s}_{1} \mathbf{u}_{2,1}, \mathbf{s}_{2} \mathbf{u}_{2,2}\right\}=\{00001111,11111000\}$ and $\mathbf{X}_{3}=$ $\left\{\mathbf{s}_{1} \mathbf{u}_{3,1}, \mathbf{s}_{2} \mathbf{u}_{3,2}\right\}=\{00000101,11110111\}$. It is easy to verify that $d_{\mathrm{S}}(\mathcal{C})=5=d_{2}$.

In Construction 1 of [14], each sequence $\mathbf{x}_{i, j}=\left(\mathbf{s}_{j}, \mathbf{u}_{i, j}\right)$ such that each $\mathbf{u}_{i, j}$ is viewed as an element of the field $\mathbb{F}_{q^{L-\lceil\log M\rceil}}$ and $\left(\mathbf{u}_{i, 1}, \cdots, \mathbf{u}_{i, M}\right)$ is a codeword of an MDS code over $\mathbb{F}_{q^{L-\lceil\log M\rceil}}$. In comparison, our construction uses codes (i.e., $\mathcal{C}_{2}$ ) of length $d_{1} M$ over $\mathbb{F}_{q}$ (rather than its extension field), and $\mathbf{u}_{i, 1}, \cdots, \mathbf{u}_{i, M}$ are obtained by dividing each codeword of $\mathcal{C}_{2}$ into $M$ segments of length $d_{1}$, which allows us to construct $\mathcal{C}_{2}$ with greater sequence-subset distance. For example, suppose $q=2, d_{1}=8 M=10$ and $N=2^{48}$. Then by Construction 1 of [14], we need a [10,6] MDS codes over the field $\mathbb{F}_{2^{8}}$, which has minimum distance 5 . In comparison, by our construction, we can let $\mathcal{C}_{2}$ be a $[80,48]$ linear code over $\mathbb{F}_{2}$ with minimum distance $d_{2}=10$ (e.g., see [25]). By 
Theorem 10, the corresponding sequence-subset code has a greater minimum distance. tion.

Construction 4 can be extended to the following construc-

Construction $4^{\prime}$ : Let $\mathcal{C}_{1}, \mathcal{C}_{2}$ and $I_{j}, j \in[M]$, be the same as in Construction 4 . Let $n$ be a given positive integer. For each $n$-tuple $\mathbf{i}=\left(i_{1}, i_{2}, \cdots, i_{n}\right) \in[N]^{n}$, let

$$
\mathbf{X}_{\mathbf{i}}=\left\{\mathbf{x}_{\mathbf{i}, 1}, \mathbf{x}_{\mathbf{i}, 2}, \cdots, \mathbf{x}_{\mathbf{i}, M}\right\}
$$

such that for each $j \in[M]$,

$$
\mathbf{x}_{\mathbf{i}, j}=\left(\mathbf{s}_{j}, \mathbf{u}_{i_{1}}\left(I_{j}\right), \cdots, \mathbf{u}_{i_{n}}\left(I_{j}\right)\right) .
$$

Finally, let

$$
\tilde{\mathcal{C}}=\left\{\mathbf{X}_{\mathbf{i}} ; \mathbf{i}=\left(i_{1}, i_{2}, \cdots, i_{n}\right) \in[N]^{n}\right\} .
$$

Then we have the following theorem.

Theorem 11: The code $\tilde{\mathcal{C}}$ obtained by Construction $4^{\prime}$ has sequence length $L=L_{1}+n d_{1}$, constant codeword size $M$ and code size $|\tilde{\mathcal{C}}|=N^{n}$, and minimum sequence-subset distance

$$
d_{\mathrm{S}}(\tilde{\mathcal{C}}) \geq d_{2}
$$

Proof: Clearly, the code $\tilde{\mathcal{C}}$ has sequence length $L=$ $L_{1}+n d_{1}$, constant codeword size $M$ and code size $|\tilde{\mathcal{C}}|=$ $N^{n}$. To estimate the minimum distance of $\tilde{\mathcal{C}}$, suppose $\left(i_{1}, i_{2}, \cdots, i_{n}\right)=\mathbf{i} \neq \mathbf{i}^{\prime}=\left(i_{1}^{\prime}, i_{2}^{\prime}, \cdots, i_{n}^{\prime}\right) \in[N]^{n}$. Without loss of generality, assume $i_{1} \neq i_{1}^{\prime}$. For each $j \in[M]$, consider the subsequence $\mathbf{x}_{\mathbf{i}, j}^{\prime}=\left(\mathbf{s}_{j}, \mathbf{u}_{i_{1}}\left(I_{j}\right)\right)$ of $\mathbf{x}_{\mathbf{i}, j}$ and the subsequence $\mathbf{x}_{\mathbf{i}^{\prime}, j}^{\prime}=\left(\mathbf{s}_{j}, \mathbf{u}_{i_{1}^{\prime}}\left(I_{j}\right)\right)$ of $\mathbf{x}_{\mathbf{i}^{\prime}, j}$. By the same discussions as in the proof of Theorem 10, we can prove that $d_{\mathrm{S}}\left(\mathbf{X}_{\mathbf{i}}^{\prime}, \mathbf{X}_{\mathbf{i}^{\prime}}^{\prime}\right) \geq d_{2}$, where $\mathbf{X}_{\mathbf{i}}^{\prime}=\left\{\mathbf{x}_{\mathbf{i}, j}^{\prime} ; j \in[M]\right\}$ and $\mathbf{X}_{\mathbf{i}^{\prime}}^{\prime}=\left\{\mathbf{x}_{\mathbf{i}^{\prime}, j}^{\prime} ; j \in[M]\right\}$. Since sequences in $\mathbf{X}_{\mathbf{i}}^{\prime}$ (resp. $\mathbf{X}_{\mathbf{i}^{\prime}}^{\prime}$ ) are subsequences of $\mathbf{X}_{\mathbf{i}}$ (resp. $\mathbf{X}_{\mathbf{i}^{\prime}}$ ), then it is easy to see that $d_{\mathrm{S}}\left(\mathbf{X}_{\mathbf{i}}, \mathbf{X}_{\mathbf{i}^{\prime}}\right) \geq d_{\mathrm{S}}\left(\mathbf{X}_{\mathbf{i}}^{\prime}, \mathbf{X}_{\mathbf{i}^{\prime}}^{\prime}\right) \geq d_{2}$. Hence, the minimum sequence-subset distance of $\tilde{\mathcal{C}}$ satisfies $d_{\mathrm{S}}(\tilde{\mathcal{C}}) \geq d_{2}$.

Example 8: Let $\mathcal{C}_{1}$ and $\mathcal{C}_{2}$ be the same as in Example 7 and let $n=2$. As in Example 7] we can denote each $\mathbf{u}_{i}$ as $\mathbf{u}_{i}=\mathbf{u}_{i, 1} \mathbf{u}_{i, 2}$. By Construction $4^{\prime}$, for each $\mathbf{i}=\left(i_{1}, i_{2}\right) \in\{1,2,3\}^{2}$, we have $\mathbf{X}_{\mathbf{i}}=$ $\left\{\mathbf{s}_{1} \mathbf{u}_{i_{1}, 1} \mathbf{u}_{i_{2}, 1}, \mathbf{s}_{2} \mathbf{u}_{i_{1}, 2} \mathbf{u}_{i_{2}, 2}\right\}$. For example, for $\mathbf{i}=(1,1)$, $\mathbf{X}_{(1,1)}=\{000000000000,111100000000\}$; for $\mathbf{i}=(1,2)$, $\mathbf{X}_{(1,2)}=\{000000001111,111100001000\}$; for $\mathbf{i}=(3,2)$, $\mathbf{X}_{(3,2)}=\{000001011111,111101111000\}$. We can estimate $d_{\mathbf{S}}\left(\mathbf{X}_{(1,2)}, \mathbf{X}_{(3,2)}\right)$ as follows. Consider $d_{\mathbf{S}}\left(\mathbf{X}_{(1,2)}^{\prime}, \mathbf{X}_{(3,2)}^{\prime}\right)$, where $\mathbf{X}_{(1,2)}^{\prime}=\left\{\mathbf{s}_{1} \mathbf{u}_{2,1}, \mathbf{s}_{2} \mathbf{u}_{2,2}\right\}=\{00000000,11110000\}$ is a subsequence of $\mathbf{X}_{(1,2)}$ and $\mathbf{X}_{(3,2)}^{\prime}=\left\{\mathbf{s}_{1} \mathbf{u}_{3,1}, \mathbf{s}_{2} \mathbf{u}_{3,2}\right\}=$ $\{00000101,11110111\}$ is a subsequence of $\mathbf{X}_{(3,2)}$. Note that $\mathbf{X}_{(1,2)}^{\prime}$ and $\mathbf{X}_{(3,2)}^{\prime}$ are two distinct codewords of the code constructed in Example 7 so we have $d_{\mathrm{S}}\left(\mathbf{X}_{(1,2)}^{\prime}, \mathbf{X}_{(3,2)}^{\prime}\right) \geq 5$, and hence $d_{\mathrm{S}}\left(\mathbf{X}_{(1,2)}, \mathbf{X}_{(3,2)}\right) \geq d_{\mathrm{S}}\left(\mathbf{X}_{(1,2)}^{\prime}, \mathbf{X}_{(3,2)}^{\prime}\right) \geq 5$. Similarly, we can verify that $d_{\mathrm{S}}\left(\mathbf{X}_{\mathbf{i}}, \mathbf{X}_{\mathbf{i}^{\prime}}\right) \geq 5$ for all distinct $\mathbf{i}, \mathbf{i}^{\prime} \in\{1,2,3\}^{2}$. Thus, the minimum sequence-subset distance of $\tilde{\mathcal{C}}$ satisfies $d_{\mathrm{S}}(\tilde{\mathcal{C}}) \geq 5=d_{2}$, where $\tilde{\mathcal{C}}=\left\{\mathbf{X}_{\mathbf{i}} ; \mathbf{i} \in\{1,2,3\}^{2}\right\}$. In fact, we have $d_{\mathrm{S}}(\tilde{\mathcal{C}})=5$ because we can verify that $d_{\mathrm{S}}\left(\mathbf{X}_{(1,2)}, \mathbf{X}_{(3,2)}\right)=5$.

\section{Conclusions And Discussions}

We introduced a new metric over the power set of the set of all vectors over a finite alphabet, which generalizes the classical Hamming distance and was used to establish a uniform framework to design error-correcting codes for DNA storage channel. Some upper bounds on the size of the sequence-subset codes were derived and some constructions of such codes were proposed.

\section{A. Open Problems in Sequence-subset Codes}

It is still an open problem to analyze the tight upper bound on the size of sequence-subset codes and design optimal codes for general parameters of sequence length, codeword size and minimum distance. Another interesting problem is how to design sequence-subset codes for DNA storage channel that can be efficiently encoded and decoded.

\section{B. Sequence-Subset Distance for Multisets}

The sequence-subset distance (Definition 1) can be directly generalized to multisets of sequences in $\mathbb{A}^{L}$. The following is an example of sequence-subset distance between multisets.

Example 9: Suppose $\mathbb{A}=\{0,1\}$ and $L=4$. Consider $\mathbf{X}_{1}=\left\{\mathbf{x}_{1}, \mathbf{x}_{2}, \mathbf{x}_{3}\right\}$ and $\mathbf{X}_{2}=\left\{\mathbf{y}_{1}, \mathbf{y}_{2}, \mathbf{y}_{3}, \mathbf{y}_{4}\right\}$, where $\mathbf{x}_{1}=$ $\mathbf{x}_{2}=0101, \mathbf{x}_{3}=1011, \mathbf{y}_{1}=0111, \mathbf{y}_{2}=1101$, and $\mathbf{y}_{3}=\mathbf{y}_{4}=$ 1001. Let $\chi_{0}: \mathbf{X}_{1} \rightarrow \mathbf{X}_{2}$ be such that $\chi_{0}\left(\mathbf{x}_{i}\right)=\mathbf{y}_{i}, i=1,2,3$. Then we can obtain $d_{\mathrm{H}}\left(\mathbf{x}_{i}, \chi_{0}\left(\mathbf{x}_{i}\right)\right)=1$ for all $i \in\{1,2,3\}$, and by (1), we have $d_{\chi_{0}}\left(\mathbf{X}_{1}, \mathbf{X}_{2}\right)=7$. Note that $d_{\mathrm{H}}\left(\mathbf{x}_{i}, \mathbf{y}_{j}\right) \geq 1$ for all $\mathbf{x}_{i} \in \mathbf{X}_{1}$ and $\mathbf{y}_{j} \in \mathbf{X}_{2}$. Then we have $d_{\chi}\left(\mathbf{X}_{1}, \mathbf{X}_{2}\right) \geq 7$ for all $\chi \in \mathscr{X}$, and hence by (2), $d_{\mathrm{S}}\left(\mathbf{X}_{1}, \mathbf{X}_{2}\right)=d_{\chi_{0}}\left(\mathbf{X}_{1}, \mathbf{X}_{2}\right)=7$.

By similar discussions as in Section II.A, we can prove that the function $d_{\mathrm{S}}(\mathbf{X}, \mathbf{Y})$ is a distance function, where $\mathbf{X}$ and $\mathbf{Y}$ are multisets of sequences in $\mathbb{A}^{L}$. Using sequencesubset distance between multisets, we can allow the output of the DNA storage channel to be multisets (rather than sets) of sequences in $\mathbb{A}^{L}$. Since in the real DNA storage, some DNA strands may have many copies that are sequenced, multisets are more suitable than sets for the output of the DNA storage channel.

Another advantage of using multisets as the output of the DNA storage channel is that it captures the case when there are $t(>1)$ strands that are changed to the same strand by substitution errors. Note that if using sets as the output of the channel, then $t-1$ of these sequences have to be viewed as lost sequences, which induces a larger sequence-subset distance between the input and output.

To study the properties of codes over the space of all multisets of $\mathbb{A}^{L}$ with sequence-subset distance is also a possible research direction.

\section{APPENDIX A}

\section{PROOF OF LEMMA 1}

If $\mathbf{X}_{1} \cap \mathbf{X}_{2}=\emptyset$, the claim is naturally true. In the following, we assume that $\mathbf{X}_{1} \cap \mathbf{X}_{2} \neq \emptyset$.

First, we claim that for each $\chi \in \mathscr{X}$ such that $d_{\mathrm{S}}\left(\mathbf{X}_{1}, \mathbf{X}_{2}\right)=$ $d_{\chi}\left(\mathbf{X}_{1}, \mathbf{X}_{2}\right)$ and each $\mathbf{y} \in \mathbf{X}_{1} \cap \mathbf{X}_{2}$, there exists an $\mathbf{x} \in \mathbf{X}_{1}$ such that $\mathbf{y}=\chi(\mathbf{x})$. This can be proved, by contradiction, as 
follows. Suppose there is a $\mathbf{y} \in \mathbf{X}_{1} \cap \mathbf{X}_{2}$ such that $\mathbf{y} \neq \chi\left(\mathbf{x}^{\prime}\right)$ for all $\mathbf{x}^{\prime} \in \mathbf{X}_{1}$. Since $\mathbf{y} \in \mathbf{X}_{1} \cap \mathbf{X}_{2}$, then we have $\chi(\mathbf{y}) \neq \mathbf{y}$, and hence we can let $\chi^{\prime}: \mathbf{X}_{1} \rightarrow \mathbf{X}_{2}$ be such that $\chi^{\prime}(\mathbf{y})=\mathbf{y}$ and $\chi^{\prime}\left(\mathbf{x}^{\prime}\right)=\chi\left(\mathbf{x}^{\prime}\right)$ for all $\mathbf{x}^{\prime} \in \mathbf{X}_{1} \backslash\{\mathbf{y}\}$ (see Fig. 2 for an illustration). Note that $d_{\mathrm{H}}\left(\mathbf{y}, \chi^{\prime}(\mathbf{y})\right)=0<d_{\mathrm{H}}(\mathbf{y}, \chi(\mathbf{y}))$ and $d_{\mathrm{H}}\left(\mathbf{x}^{\prime}, \chi^{\prime}\left(\mathbf{x}^{\prime}\right)\right)=d_{\mathrm{H}}\left(\mathbf{x}^{\prime}, \chi\left(\mathbf{x}^{\prime}\right)\right)$ for all $\mathbf{x}^{\prime} \in \mathbf{X}_{1} \backslash\{\mathbf{y}\}$. By $1 \mathbf{1}$, we have $d_{\chi^{\prime}}\left(\mathbf{X}_{1}, \mathbf{X}_{2}\right)<d_{\chi}\left(\mathbf{X}_{1}, \mathbf{X}_{2}\right)$, which contradicts to (2). Hence, by contradiction, for each $\mathbf{y} \in \mathbf{X}_{1} \cap \mathbf{X}_{2}$, there exists an $\mathbf{x} \in \mathbf{X}_{1}$ such that $\mathbf{y}=\chi(\mathbf{x})$.

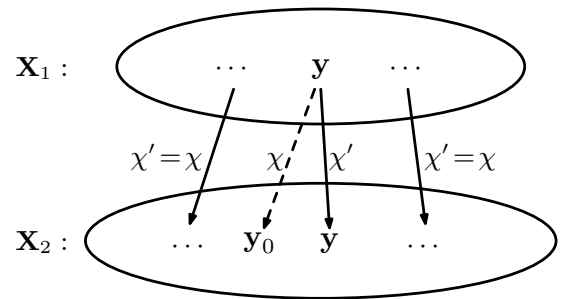

Fig 2. An illustration of the injections in the proof of Lemma 1 For the injection $\chi$, there exists a $\mathbf{y} \in \mathbf{X}_{1} \cap \mathbf{X}_{2}$ such that $\chi(\mathbf{y}) \neq \mathbf{y}$. Denote $\chi(\mathbf{y})=\mathbf{y}_{0}$. Then we can modify the injection $\chi$ to a different injection $\chi^{\prime}$ by letting $\chi^{\prime}(\mathbf{y})=\mathbf{y}$, and the image of all other elements of $\mathbf{X}_{1}$ keep unchanged.

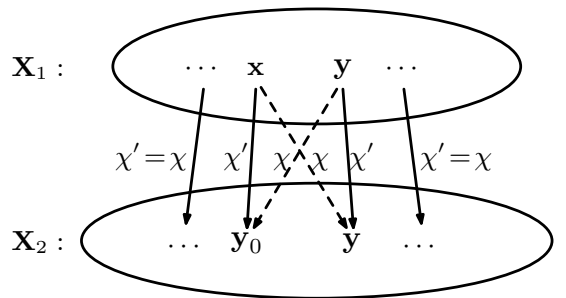

Fig 3. An illustration of the bijections in the proof of Lemma 1 For the bijection $\chi$, we have $\chi(\mathbf{x})=\mathbf{y}$ and $\chi(\mathbf{y})=\mathbf{y}_{0} \neq \mathbf{y}$, where $\mathbf{y} \in \mathbf{X}_{1} \cap \mathbf{X}_{2}$. We modify the bijection $\chi$ to a different bijection $\chi^{\prime}$ by letting $\chi^{\prime}(\mathbf{x})=\mathbf{y}_{0}$ and $\chi^{\prime}(\mathbf{y})=\mathbf{y}$, and the image of all other elements of $\mathbf{X}_{1}$ keeping unchanged.

Now, pick a $\chi \in \mathscr{X}$ such that $d_{\mathrm{S}}\left(\mathbf{X}_{1}, \mathbf{X}_{2}\right)=d_{\chi}\left(\mathbf{X}_{1}, \mathbf{X}_{2}\right)$ and denote

$$
\mathcal{N}(\chi)=\left\{\mathbf{y}^{\prime} \in \mathbf{X}_{1} \cap \mathbf{X}_{2} ; \chi\left(\mathbf{y}^{\prime}\right) \neq \mathbf{y}^{\prime}\right\} .
$$

If $\mathcal{N}(\chi)=\emptyset$, then by the definition of $\mathcal{N}(\chi), \chi(\mathbf{x})=\mathbf{x}$ for all $\mathbf{x} \in \mathbf{X}_{1} \cap \mathbf{X}_{2}$ and we can choose $\chi_{0}=\chi$. Otherwise, pick a $\mathbf{y} \in \mathcal{N}(\chi)$ and we have $\chi(\mathbf{y})=\mathbf{y}_{0}$ for some $\mathbf{y}_{0} \in \mathbf{X}_{2} \backslash\{\mathbf{y}\}$. Moreover, by previous discussion, there exists an $\mathbf{x} \in \mathbf{X}_{1}$ such that $\mathbf{y}=\chi(\mathbf{x})$. Then we can let $\chi^{\prime}: \mathbf{X}_{1} \rightarrow \mathbf{X}_{2}$ be such that $\chi^{\prime}(\mathbf{x})=\mathbf{y}_{0}, \chi^{\prime}(\mathbf{y})=\mathbf{y}$ and $\chi^{\prime}\left(\mathbf{x}^{\prime}\right)=\chi_{0}\left(\mathbf{x}^{\prime}\right)$ for all $\mathbf{x}^{\prime} \in$ $\mathbf{X}_{1} \backslash\{\mathbf{x}, \mathbf{y}\}$ (see Fig. 3 for an illustration). Note that

$$
\begin{aligned}
d_{\mathrm{H}}\left(\mathbf{x}, \chi^{\prime}(\mathbf{x})\right)+d_{\mathrm{H}}\left(\mathbf{y}, \chi^{\prime}(\mathbf{y})\right) & =d_{\mathrm{H}}\left(\mathbf{x}, \mathbf{y}_{0}\right)+d_{\mathrm{H}}(\mathbf{y}, \mathbf{y}) \\
& =d_{\mathrm{H}}\left(\mathbf{x}, \mathbf{y}_{0}\right) \\
& \leq d_{\mathrm{H}}(\mathbf{x}, \mathbf{y})+d_{\mathrm{H}}\left(\mathbf{y}, \mathbf{y}_{0}\right) \\
& =d_{\mathrm{H}}(\mathbf{x}, \chi(\mathbf{x}))+d_{\mathrm{H}}(\mathbf{y}, \chi(\mathbf{y}))
\end{aligned}
$$

and by construction of $\chi^{\prime}$,

$$
d_{\mathrm{H}}\left(\mathbf{x}^{\prime}, \chi^{\prime}\left(\mathbf{x}^{\prime}\right)\right)=d_{\mathrm{H}}\left(\mathbf{x}^{\prime}, \chi\left(\mathbf{x}^{\prime}\right)\right), \forall \mathbf{x}^{\prime} \in \mathbf{X}_{1} \backslash\{\mathbf{x}, \mathbf{y}\} .
$$

By (2), we have

$$
d_{\mathrm{S}}\left(\mathbf{X}_{1}, \mathbf{X}_{2}\right)=d_{\chi}\left(\mathbf{X}_{1}, \mathbf{X}_{2}\right)=d_{\chi^{\prime}}\left(\mathbf{X}_{1}, \mathbf{X}_{2}\right) .
$$

Again by construction of $\chi^{\prime}$, we have $\mathcal{N}\left(\chi^{\prime}\right)=\mathcal{N}(\chi) \backslash\{\mathbf{y}\}$, and hence

$$
\left|\mathcal{N}\left(\chi^{\prime}\right)\right|=|\mathcal{N}(\chi)|-1,
$$

where

$$
\mathcal{N}\left(\chi^{\prime}\right)=\left\{\mathbf{y} \in \mathbf{X}_{1} \cap \mathbf{X}_{2} ; \chi^{\prime}(\mathbf{y}) \neq \mathbf{y}\right\} .
$$

If $\mathcal{N}\left(\chi^{\prime}\right)=\emptyset$, then $\chi^{\prime}(\mathbf{x})=\mathbf{x}$ for all $\mathbf{x} \in \mathbf{X}_{1} \cap \mathbf{X}_{2}$ and we can choose $\chi_{0}=\chi^{\prime}$. Otherwise, by the same discussion, we can obtain a $\chi^{\prime \prime}: \mathbf{X}_{1} \rightarrow \mathbf{X}_{2}$ such that $d_{\mathrm{S}}\left(\mathbf{X}_{1}, \mathbf{X}_{2}\right)=d_{\chi^{\prime \prime}}\left(\mathbf{X}_{1}, \mathbf{X}_{2}\right)$ and $\left|\mathcal{N}\left(\chi^{\prime \prime}\right)\right|=\left|\mathcal{N}\left(\chi^{\prime}\right)\right|-1$, and so on. Noting that $\mathcal{N}\left(\chi^{\prime \prime}\right) \subseteq$ $\mathbf{X}_{1} \cap \mathbf{X}_{2}$ is a finite set, we can always find a $\chi_{0} \in \mathscr{X}$ such that $d_{\mathrm{S}}\left(\mathbf{X}_{1}, \mathbf{X}_{2}\right)=d_{\chi_{0}}\left(\mathbf{X}_{1}, \mathbf{X}_{2}\right)$ and

$$
\mathcal{N}\left(\chi_{0}\right)=\left\{\mathbf{y} \in \mathbf{X}_{1} \cap \mathbf{X}_{2} ; \chi_{0}(\mathbf{y}) \neq \mathbf{y}\right\}=\emptyset .
$$

Hence, we have $\chi_{0}(\mathbf{x})=\mathbf{x}$ for all $\mathbf{x} \in \mathbf{X}_{1} \cap \mathbf{X}_{2}$, which completes the proof.

\section{APPENDiX B}

\section{PROOF OF LEMMA 2}

It suffices to prove that if $\mathbf{X}_{2}^{\prime} \subseteq \mathbf{X}_{2}$ and $\left|\mathbf{X}_{1}\right| \leq\left|\mathbf{X}_{2}^{\prime}\right|=$ $\left|\mathbf{X}_{2}\right|-1$, then

$$
d_{\mathrm{S}}\left(\mathbf{X}_{1}, \mathbf{X}_{2}^{\prime}\right) \leq d_{\mathrm{S}}\left(\mathbf{X}_{1}, \mathbf{X}_{2}\right) .
$$

Without loss of generality, we can assume

$$
\begin{aligned}
& \mathbf{X}_{1}=\left\{\mathbf{x}_{1}, \cdots, \mathbf{x}_{n}\right\}, \\
& \mathbf{X}_{2}^{\prime}=\left\{\mathbf{y}_{1}, \cdots, \mathbf{y}_{n}, \mathbf{y}_{n+1}, \cdots, \mathbf{y}_{n+s-1}\right\}
\end{aligned}
$$

and

$$
\mathbf{X}_{2}=\left\{\mathbf{y}_{1}, \cdots, \mathbf{y}_{n}, \mathbf{y}_{n+1}, \cdots, \mathbf{y}_{n+s-1}, \mathbf{y}_{n+s}\right\},
$$

where $s \geq 1$, such that

$$
d_{\mathrm{S}}\left(\mathbf{X}_{1}, \mathbf{X}_{2}^{\prime}\right)=\sum_{i=1}^{n} d_{\mathrm{H}}\left(\mathbf{x}_{i}, \mathbf{y}_{i}\right)+L(s-1) .
$$

By Definition 1 we can suppose

$$
d_{\mathrm{S}}\left(\mathbf{X}_{1}, \mathbf{X}_{2}\right)=\sum_{i=1}^{n} d_{\mathrm{H}}\left(\mathbf{x}_{i}, \mathbf{y}_{\ell_{i}}\right)+L s,
$$

where $\left\{\ell_{i} ; i=1,2, \cdots, n\right\}$ is a subset of $\{1,2, \cdots, n+s\}$. We have the following two cases.

Case 1: $n+s \notin\left\{\ell_{1}, \ell_{2}, \cdots, \ell_{n}\right\}$. In this case, we have

$$
\begin{aligned}
d_{\mathrm{S}}\left(\mathbf{X}_{1}, \mathbf{X}_{2}^{\prime}\right) & =\sum_{i=1}^{n} d_{\mathrm{H}}\left(\mathbf{x}_{i}, \mathbf{y}_{i}\right)+L(s-1) \\
& \leq \sum_{i=1}^{n} d_{\mathrm{H}}\left(\mathbf{x}_{i}, \mathbf{y}_{\ell_{i}}\right)+L(s-1) \\
& <\sum_{i=1}^{n}\left(d_{\mathrm{H}}\left(\mathbf{x}_{i}, \mathbf{y}_{\ell_{i}}\right)+L s\right. \\
& =d_{\mathrm{S}}\left(\mathbf{X}_{1}, \mathbf{X}_{2}\right),
\end{aligned}
$$

where the first inequality is obtained by (2).

Case 2: There exists a $k \in\{1,2, \cdots, n\}$ such that $n+s=\ell_{k}$. Noticing that $s \geq 1$, then there exists an $m \in\{1,2, \cdots, n+s-1\}$ such that $m \notin\left\{\ell_{1}, \ell_{2}, \cdots, \ell_{n}\right\}$. 
Denote $\ell_{k}^{\prime}=m$ and $\ell_{i}^{\prime}=\ell_{i}$ for $i \in\{1,2, \cdots, n\} \backslash\{k\}$. Then we have

$$
\left\{\ell_{1}^{\prime}, \ell_{2}^{\prime}, \cdots, \ell_{n}^{\prime}\right\} \subseteq\{1,2, \cdots, n+s-1\} .
$$

Moreover, noticing that $\left\{\mathbf{x}_{k}, \mathbf{y}_{m}, \mathbf{y}_{\ell_{k}}\right\} \subseteq \mathbf{X}_{1} \cup \mathbf{X}_{2} \subseteq \mathbb{A}^{L}$, then $d_{\mathrm{H}}\left(\mathbf{x}_{k}, \mathbf{y}_{m}\right) \leq L$ and $d_{\mathrm{H}}\left(\mathbf{x}_{k}, \mathbf{y}_{\ell_{k}}\right) \leq L$. Hence, we can obtain

$$
d_{\mathrm{H}}\left(\mathbf{x}_{k}, \mathbf{y}_{m}\right)-d_{\mathrm{H}}\left(\mathbf{x}_{k}, \mathbf{y}_{\ell_{k}}\right) \leq L
$$

And further we have

$$
\begin{aligned}
d_{\mathrm{S}}\left(\mathbf{X}_{1}, \mathbf{X}_{2}^{\prime}\right)= & \sum_{i=1}^{n} d_{\mathrm{H}}\left(\mathbf{x}_{i}, \mathbf{y}_{i}\right)+L(s-1) \\
\leq & \sum_{i=1}^{n} d_{\mathrm{H}}\left(\mathbf{x}_{i}, \mathbf{y}_{\ell_{i}^{\prime}}\right)+L(s-1) \\
= & \sum_{i=1}^{n} d_{\mathrm{H}}\left(\mathbf{x}_{i}, \mathbf{y}_{\ell_{i}}\right)-d_{\mathrm{H}}\left(\mathbf{x}_{k}, \mathbf{y}_{\ell_{k}}\right)+d_{\mathrm{H}}\left(\mathbf{x}_{k}, \mathbf{y}_{m}\right) \\
& +L(s-1) \\
\leq & \sum_{i=1}^{n}\left(d_{\mathrm{H}}\left(\mathbf{x}_{i}, \mathbf{y}_{\ell_{i}}\right)+L+L(s-1)\right. \\
= & d_{\mathrm{S}}\left(\mathbf{X}_{1}, \mathbf{X}_{2}\right)
\end{aligned}
$$

where the first inequality is obtained by (27) and (2), and the second inequality is obtained by (28).

Thus, we always have $d_{\mathrm{S}}\left(\mathbf{X}_{1}, \mathbf{X}_{2}^{\prime}\right) \leq d_{\mathrm{S}}\left(\mathbf{X}_{1}, \mathbf{X}_{2}\right)$, which completes the proof.

\section{APPENDIX C \\ PROOF OF THEOREM 1}

By Definition 1, it is easy to see that for any two subsets $\mathbf{X}_{1}$ and $\mathbf{X}_{2}$ of $\mathbb{A}^{L}, d_{\mathrm{S}}\left(\mathbf{X}_{1}, \mathbf{X}_{2}\right)=d_{\mathrm{S}}\left(\mathbf{X}_{2}, \mathbf{X}_{1}\right) \geq 0$. Moreover, by Corollary 1, we can easily see that $d_{\mathrm{S}}\left(\mathbf{X}_{1}, \mathbf{X}_{2}\right)=0$ if and only if $\mathbf{X}_{1}=\mathbf{X}_{2}$. To prove that $d_{\mathrm{S}}(\cdot, \cdot)$ is a distance function, we only need to prove the triangle inequality, that is,

$$
d_{\mathrm{S}}\left(\mathbf{X}_{1}, \mathbf{X}_{2}\right) \leq d_{\mathrm{S}}\left(\mathbf{X}_{1}, \mathbf{X}_{3}\right)+d_{\mathrm{S}}\left(\mathbf{X}_{2}, \mathbf{X}_{3}\right)
$$

for any three subsets $\mathbf{X}_{1}, \mathbf{X}_{2}$ and $\mathbf{X}_{3}$ of $\mathbb{A}^{L}$. Without loss of generality, we can assume that $\left|\mathbf{X}_{1}\right| \leq\left|\mathbf{X}_{2}\right|$. Then we have the following three cases.

Case 1. $\left|\mathbf{X}_{1}\right| \leq\left|\mathbf{X}_{2}\right| \leq\left|\mathbf{X}_{3}\right|$. In this case, we can fix a subset $\mathbf{X}_{3}^{\prime} \subseteq \mathbf{X}_{3}$ of size $\left|\mathbf{X}_{3}^{\prime}\right|=\left|\mathbf{X}_{2}\right|$. Then by Lemma 2 $d_{\mathrm{S}}\left(\mathbf{X}_{1}, \mathbf{X}_{3}^{\prime}\right) \leq d_{\mathrm{S}}\left(\mathbf{X}_{1}, \mathbf{X}_{3}\right)$ and $d_{\mathrm{S}}\left(\mathbf{X}_{2}, \mathbf{X}_{3}^{\prime}\right) \leq d_{\mathrm{S}}\left(\mathbf{X}_{2}, \mathbf{X}_{3}\right)$. It suffices to prove that

$$
d_{\mathrm{S}}\left(\mathbf{X}_{1}, \mathbf{X}_{2}\right) \leq d_{\mathrm{S}}\left(\mathbf{X}_{1}, \mathbf{X}_{3}^{\prime}\right)+d_{\mathrm{S}}\left(\mathbf{X}_{2}, \mathbf{X}_{3}^{\prime}\right)
$$

Without loss of generality, we can assume

$$
\begin{aligned}
& \mathbf{X}_{1}=\left\{\mathbf{x}_{1}, \cdots, \mathbf{x}_{n}\right\} \\
& \mathbf{X}_{2}=\left\{\mathbf{y}_{1}, \cdots, \mathbf{y}_{n}, \mathbf{y}_{n+1}, \cdots, \mathbf{y}_{n+s}\right\} \\
& \mathbf{X}_{3}^{\prime}=\left\{\mathbf{z}_{1}, \cdots, \mathbf{z}_{n}, \mathbf{z}_{n+1}, \cdots, \mathbf{z}_{n+s}\right\}
\end{aligned}
$$

such that

$$
d_{\mathrm{S}}\left(\mathbf{X}_{1}, \mathbf{X}_{3}^{\prime}\right)=\sum_{i=1}^{n} d_{\mathrm{H}}\left(\mathbf{x}_{i}, \mathbf{z}_{i}\right)+L s
$$

$$
d_{\mathrm{S}}\left(\mathbf{X}_{2}, \mathbf{X}_{3}^{\prime}\right)=\sum_{i=1}^{n+s} d_{\mathrm{H}}\left(\mathbf{y}_{i}, \mathbf{z}_{i}\right)
$$

and

$$
d_{\mathrm{S}}\left(\mathbf{X}_{1}, \mathbf{X}_{2}\right)=\sum_{i=1}^{n} d_{\mathrm{H}}\left(\mathbf{x}_{i}, \mathbf{y}_{\ell_{i}}\right)+L s
$$

where $s \geq 0$ and $\left\{\ell_{1}, \ell_{2}, \cdots, \ell_{n}\right\} \subseteq\{1,2, \cdots, n+s\}$. Then we have

$$
\begin{aligned}
d_{\mathrm{S}}\left(\mathbf{X}_{1}, \mathbf{X}_{2}\right) & =\sum_{i=1}^{n} d_{\mathrm{H}}\left(\mathbf{x}_{i}, \mathbf{y}_{\ell_{i}}\right)+L s \\
& \leq \sum_{i=1}^{n} d_{\mathrm{H}}\left(\mathbf{x}_{i}, \mathbf{y}_{i}\right)+L s \\
& \leq \sum_{i=1}^{n}\left(d_{\mathrm{H}}\left(\mathbf{x}_{i}, \mathbf{z}_{i}\right)+d_{\mathrm{H}}\left(\mathbf{y}_{i}, \mathbf{z}_{i}\right)\right)+L s \\
& \leq \sum_{i=1}^{n} d_{\mathrm{H}}\left(\mathbf{x}_{i}, \mathbf{z}_{i}\right)+L s+\sum_{i=1}^{n+s} d_{\mathrm{H}}\left(\mathbf{y}_{i}, \mathbf{z}_{i}\right) \\
& =d_{\mathrm{S}}\left(\mathbf{X}_{1}, \mathbf{X}_{3}^{\prime}\right)+d_{\mathrm{S}}\left(\mathbf{X}_{2}, \mathbf{X}_{3}^{\prime}\right) \\
& \leq d_{\mathrm{S}}\left(\mathbf{X}_{1}, \mathbf{X}_{3}\right)+d_{\mathrm{S}}\left(\mathbf{X}_{2}, \mathbf{X}_{3}\right),
\end{aligned}
$$

where the first inequality is obtained by (2) and the last inequality is obtained by Lemma 2

Case 2. $\left|\mathbf{X}_{1}\right| \leq\left|\mathbf{X}_{3}\right| \leq\left|\mathbf{X}_{2}\right|$. In this case, we can assume

$$
\begin{aligned}
& \mathbf{X}_{1}=\left\{\mathbf{x}_{1}, \cdots, \mathbf{x}_{n}\right\} \\
& \mathbf{X}_{3}=\left\{\mathbf{y}_{1}, \cdots, \mathbf{y}_{n}, \mathbf{y}_{n+1}, \cdots, \mathbf{y}_{n+s}\right\} \\
& \mathbf{X}_{2}=\left\{\mathbf{z}_{1}, \cdots, \mathbf{z}_{n}, \mathbf{z}_{n+1}, \cdots, \mathbf{z}_{n+s}, \mathbf{z}_{n+s+1}, \cdots, \mathbf{z}_{n+s+t}\right\}
\end{aligned}
$$

such that

$$
\begin{aligned}
& d_{\mathrm{S}}\left(\mathbf{X}_{1}, \mathbf{X}_{3}\right)=\sum_{i=1}^{n} d_{\mathrm{H}}\left(\mathbf{x}_{i}, \mathbf{y}_{i}\right)+L s \\
& d_{\mathrm{S}}\left(\mathbf{X}_{2}, \mathbf{X}_{3}\right)=\sum_{i=1}^{n+s} d_{\mathrm{H}}\left(\mathbf{y}_{i}, \mathbf{z}_{i}\right)+L t
\end{aligned}
$$

and

$$
d_{\mathrm{S}}\left(\mathbf{X}_{1}, \mathbf{X}_{2}\right)=\sum_{i=1}^{n} d_{\mathrm{H}}\left(\mathbf{x}_{i}, \mathbf{z}_{\ell_{i}}\right)+L(s+t),
$$

where $s, t \geq 0$ and $\left\{\ell_{1}, \ell_{2}, \cdots, \ell_{n}\right\} \subseteq\{1,2, \cdots, n+s+t\}$. Then we have

$$
\begin{aligned}
d_{\mathrm{S}}\left(\mathbf{X}_{1}, \mathbf{X}_{2}\right) & =\sum_{i=1}^{n} d_{\mathrm{H}}\left(\mathbf{x}_{i}, \mathbf{z}_{\ell_{i}}\right)+L(s+t) \\
& \leq \sum_{i=1}^{n} d_{\mathrm{H}}\left(\mathbf{x}_{i}, \mathbf{z}_{i}\right)+L(s+t) \\
& \leq \sum_{i=1}^{n}\left(d_{\mathrm{H}}\left(\mathbf{x}_{i}, \mathbf{y}_{i}\right)+d_{\mathrm{H}}\left(\mathbf{y}_{i}, \mathbf{z}_{i}\right)\right)+L(s+t) \\
& \leq \sum_{i=1}^{n} d_{\mathrm{H}}\left(\mathbf{x}_{i}, \mathbf{y}_{i}\right)+L s+\sum_{i=1}^{n+s} d_{\mathrm{H}}\left(\mathbf{y}_{i}, \mathbf{z}_{i}\right)+L t \\
& =d_{\mathrm{S}}\left(\mathbf{X}_{1}, \mathbf{X}_{3}\right)+d_{\mathrm{S}}\left(\mathbf{X}_{2}, \mathbf{X}_{3}\right)
\end{aligned}
$$

where the first inequality is obtained by (2). 
Case 3. $\left|\mathbf{X}_{3}\right| \leq\left|\mathbf{X}_{1}\right| \leq\left|\mathbf{X}_{2}\right|$. In this case, we can assume

$$
\begin{aligned}
& \mathbf{X}_{3}=\left\{\mathbf{x}_{1}, \cdots, \mathbf{x}_{n}\right\}, \\
& \mathbf{X}_{1}=\left\{\mathbf{y}_{1}, \cdots, \mathbf{y}_{n}, \mathbf{y}_{n+1}, \cdots, \mathbf{y}_{n+s}\right\} \\
& \mathbf{X}_{2}=\left\{\mathbf{z}_{1}, \cdots, \mathbf{z}_{n}, \mathbf{z}_{n+1}, \cdots, \mathbf{z}_{n+s}, \mathbf{z}_{n+s+1}, \cdots, \mathbf{z}_{n+s+t}\right\}
\end{aligned}
$$

such that

$$
\begin{gathered}
d_{\mathrm{S}}\left(\mathbf{X}_{1}, \mathbf{X}_{3}\right)=\sum_{i=1}^{n} d_{\mathrm{H}}\left(\mathbf{x}_{i}, \mathbf{y}_{i}\right)+L s, \\
d_{\mathrm{S}}\left(\mathbf{X}_{2}, \mathbf{X}_{3}\right)=\sum_{i=1}^{n} d_{\mathrm{H}}\left(\mathbf{x}_{i}, \mathbf{z}_{i}\right)+L(s+t)
\end{gathered}
$$

and

$$
d_{\mathrm{S}}\left(\mathbf{X}_{1}, \mathbf{X}_{2}\right)=\sum_{i=1}^{n+s} d_{\mathrm{H}}\left(\mathbf{y}_{i}, \mathbf{z}_{\ell_{i}}\right)+L t
$$

where $s, t \geq 0$ and $\left\{\ell_{1}, \ell_{2}, \cdots, \ell_{n}\right\} \subseteq\{1,2, \cdots, n+s+t\}$. Then we have

$$
\begin{aligned}
d_{\mathrm{S}}\left(\mathbf{X}_{1}, \mathbf{X}_{2}\right) & =\sum_{i=1}^{n+s} d_{\mathrm{H}}\left(\mathbf{y}_{i}, \mathbf{z}_{\ell_{i}}\right)+L t \\
& \leq \sum_{i=1}^{n+s} d_{\mathrm{H}}\left(\mathbf{y}_{i}, \mathbf{z}_{i}\right)+L t \\
& \leq \sum_{i=1}^{n+s}\left(d_{\mathrm{H}}\left(\mathbf{x}_{i}, \mathbf{y}_{i}\right)+d_{\mathrm{H}}\left(\mathbf{x}_{i}, \mathbf{z}_{i}\right)\right)+L t \\
& \leq \sum_{i=1}^{n} d_{\mathrm{H}}\left(\mathbf{x}_{i}, \mathbf{y}_{i}\right)+L s+\sum_{i=1}^{n} d_{\mathrm{H}}\left(\mathbf{x}_{i}, \mathbf{z}_{i}\right)+L(s+t) \\
& =d_{\mathrm{S}}\left(\mathbf{X}_{1}, \mathbf{X}_{3}\right)+d_{\mathrm{S}}\left(\mathbf{X}_{2}, \mathbf{X}_{3}\right),
\end{aligned}
$$

where the first inequality is obtained by (2), and the third inequality is obtained from the simple fact that $d_{\mathrm{H}}(\cdot, \cdot) \leq L$.

For all cases, we have proved that

$$
d_{\mathrm{S}}\left(\mathbf{X}_{1}, \mathbf{X}_{2}\right) \leq d_{\mathrm{S}}\left(\mathbf{X}_{1}, \mathbf{X}_{3}\right)+d_{\mathrm{S}}\left(\mathbf{X}_{2}, \mathbf{X}_{3}\right) .
$$

Hence, $d_{\mathrm{S}}(\cdot, \cdot)$ satisfies the triangle inequality.

By the above discussion, we proved that $d_{\mathrm{S}}(\cdot, \cdot)$ is a distance function over $\mathcal{P}\left(\mathbb{A}^{L}\right)$.

\section{REFERENCES}

[1] J. Davis, "Microvenus," Art J, 55, 70 (1996), doi:10.2307/777811

[2] G. M. Church, Y. Gao, and S. Kosuri, "Next-generation digital information storage in DNA," Science, vol. 337, no. 6102, pp. 1628-1628, 2012.

[3] L. Organick, S. D. Ang, Y. J. Chen, R. Lopez, S. Yekhanin, K Makarychev, M. Z. Racz, G. Kamath, P. Gopalan, B. Nguyen, C. Takahashi, S. Newman, H. Y. Parker, C. Rashtchian, G. G. K. Stewart, R. Carlson, J. Mulligan, D. Carmean, G. Seelig, L. Ceze, and K. Strauss, "Random access in large-scale DNA data storage," Nature Biotechnology, vol. 36, pp. 242-248, Feb. 2018

[4] C. Rashtchian, K. Makarychev, M. Racz, S. Ang, D. Jevdjic, S. Yekhanin, L. Ceze, and K. Strauss, "Clustering billions of reads for DNA data storage," NIPS, 2017.

[5] V. I. Levenshtein, "Efficient reconstruction of sequences," IEEE Trans. on Inform. Theory, vol. 47, no. 1, pp. 2-22, Jan. 2001.

[6] N. Goldman, P. Bertone, S. Chen, C. Dessimoz, E. M. LeProust, B Sipos, and E. Birney, "Towards practical, high-capacity, lowmaintenance information storage in synthesized DNA," Nature, vol. 494, no. 7435, pp. 77-80, 2013.

[7] R. N. Grass, R. Heckel, M. Puddu, D. Paunescu, and W. J. Stark, "Robust chemical preservation of digital information on DNA in silica with errorcorrecting codes," Angew. Chem. Int. Ed., vol. 54, no. 8, pp. 2552-2555, 2015.
[8] M. Blawat, K. Gaedke1, I. Hütter, X.-M. Chen, B. Turczyk, S. Inverso, B. W. Pruitt, G. M. Church, "Forward error correction for DNA data storage," Procedia Compu Sci, vol. 80, pp. 1011-1022, 2016.

[9] S. M. H. T. Yazdi, Y. Yuan, J. Ma, H. Zhao, and O. Milenkovic, "A Rewritable, Random-Access DNA-Based Storage System," Nature Scientific Reports, 5(14138), 2015.

[10] J. Bornholt, R. Lopez, D. M. Carmean, L. Ceze, G. Seelig, and K. Strauss, "A DNA-based archival storage system," in Proceedings of the Twenty-First International Conference on Architectural Support for Programming Languages and Operating Systems, ACM, pp. 637-649, 2016.

[11] Y. Erlich, and D. Zielinski, "DNA Fountain enables a robust and efficient storage architecture," Science, vol. 355, no. 6328, pp. 950-954, 2017.

[12] W. Song, K. Cai, M. Zhang, and C. Yuen , "Codes with Run-Length and GC-Content Constraints for DNA-based Data Storage," IEEE Communications Letters, 2018, DOI: 10.1109/LCOMM.2018.2866566

[13] K.A.S. Immink, and K. Cai, "Design of Capacity-Approaching Constrained Codes for DNA-Based Storage Systems," IEEE Communications Letters, vol. 22, no. 2, pp. 224-227, 2018.

[14] A. Lenz, P. H. Siegel, A. W-Zeh, and E. Yaakobi, "Coding over Sets for DNA Storage," in Proc. IEEE Int. Symp. Inform. Theory (ISIT), 2019, pp. 2411-2415.

[15] J. Sima, N. Raviv, and J. Bruck, "On Coding over Sliced Information," 2018, Available: https://arxiv.org/abs/1809.02716

[16] R. Heckel, I. Shomorony, K. Ramchandran, and D. N. C. Tse, "Fundamental limits of DNA storage systems," in IEEE Int. Symp. Inform. Theory (ISIT), Aachen, Germany, Jun. 2017, pp. 3130-3134.

[17] H. M. Kiah, G. J. Puleo, and O. Milenkovic, "Codes for DNA sequence profiles," IEEE Trans. Inf. Theory, vol. 62, no. 6, pp. 3125-3146, Jun. 2016.

[18] M. Langberg, M. Schwartz, and E. Yaakobi, "Coding for the $\ell_{\infty}$-Limited Permutation Channel," IEEE Trans. Inf. Theory, vol. 63, no. 12, pp. 76767686, Dec. 2017.

[19] M. Kovačević, and V. Y. F. Tan, "Codes in the Space of Multisets Coding for Permutation Channels with Impairments," IEEE Trans. Inf. Theory, 2018, DOI: 10.1109/TIT.2017.2789292

[20] M. Plotkin, "Binary codes with specified minimum distances," IEEE Trans. Inf. Theory, vol. IT-6, pp. 445-450, 1960.

[21] S. M. Johnson, "A new upper bound for error-correcting codes," IEEE Trans. Inf. Theory, vol. IT-8, pp. 203-207, 1962.

[22] J.Munkres, "Algorithms for the assignment and transportation problems," J. Soc. Ind. Appl. Math., vol. 5, no. 1, pp. 32-38, Mar. 1957.

[23] W. C. Huffman and V. Pless, Fundamentals of Error-Correcting Codes. Cambridge, U.K.: Cambridge Univ. Press, 2003

[24] S. Jukna, Extremal Combinatorics. New York: Springer-Verlag, 2001.

[25] M. Grassl, "Bounds on the minimum distance of linear codes and quantum codes," Online available at http://www.codetables.de Accessed on 2019-11-13. 